\title{
Computing the Homology of Semialgebraic Sets. II: General formulas*
}

\author{
Peter Bürgisser $^{\dagger}$ \\ Technische Universität Berlin \\ Institut für Mathematik \\ GERMANY \\ pbuerg@math.tu-berlin.de
}

\author{
Felipe Cucker ${ }^{\ddagger}$ \\ Dept. of Mathematics \\ City University of Hong Kong \\ HONG KONG \\ macucker@cityu.edu.hk
}

\author{
Josué Tonelli-Cueto ${ }^{\S}$ \\ Inria Paris \& IMJ-PRG \\ OURAGAN team \\ Sorbonne Université \\ Paris, FRANCE \\ josue.tonelli.cueto@bizkaia.eu
}

\begin{abstract}
We describe and analyze a numerical algorithm for computing the homology (Betti numbers and torsion coefficients) of semialgebraic sets given by Boolean formulas. The algorithm works in weak exponential time. This means that outside a subset of data having exponentially small measure, the cost of the algorithm is single exponential in the size of the data. This extends the work in 2 to arbitrary semialgebraic sets.

All previous algorithms proposed for this problem have doubly exponential complexity.
\end{abstract}

\section{Introduction}

This paper is a continuation of [2]. In the latter, we exhibited a numerical algorithm computing the topology of a closed semialgebraic set described by a monotone Boolean combination of polynomial equalities and lax inequalities. This restriction, that the formula defining the Boolean combination is lax, forces connected components of the (projective closure of the) semialgebraic set to be separated by a positive distance. The fact that we can control these distances by the condition number of the tuple of polynomials in the description of the set was central in the design of the algorithm.

Our goal in this paper is to exhibit a numerical algorithm, with similar complexity bounds to that in [2], but working for arbitrary semialgebraic sets. That is, complements are

\footnotetext{
${ }^{*}$ This work was supported by the Einstein Foundation, Berlin.

${ }^{\dagger}$ Partially funded by the European Research Council (ERC) under the European's Horizon 2020 research and innovation programme (grant agreement No 787840).

${ }^{\ddagger}$ Partially supported by a GRF grant from the Research Grants Council of the Hong Kong SAR (project number CityU 11302418).

${ }^{\S}$ Partially supported by ANR JCJC GALOP (ANR-17-CE40-0009), the PGMO grant ALMA, and the PHC GRAPE.
} 
allowed in the Boolean combinations (or, equivalently, negations in the Boolean formulas) describing semialgebraic sets and so are strict inequalities. This algorithm works in weak exponential time. This means that its cost, or running time, is single exponential outside an exceptional set whose measure vanishes exponentially fast. Outside this exceptional set then, our algorithm works exponentially faster than state-of-the-art algorithms (which are doubly exponential). For a background on numerical algorithms, condition, and weak cost - and to avoid being repetitious - we refer the reader to the introduction of [2]. We thus proceed with a description of the problem and our main result.

In all what follows we fix natural numbers $q \geq 1, n \geq 2$ and a tuple $\boldsymbol{d}:=\left(d_{1}, \ldots, d_{q}\right) \in$ $\mathbb{N}^{q}$. We denote by $\mathcal{P}_{\boldsymbol{d}}[q]$ the linear space of polynomial tuples $p=\left(p_{1}, \ldots, p_{q}\right)$ with $p_{i} \in$ $\mathbb{R}\left[X_{1}, \ldots, X_{n}\right]$ of degree at most $d_{i}$. We let $D:=\max \left\{d_{1}, \ldots, d_{q}\right\}$ and $N:=\operatorname{dim} \mathcal{P}_{\boldsymbol{d}}[q]$. We consider the latter to be the size of the elements in $\mathcal{P}_{\boldsymbol{d}}[q]$ as this is the number of real numbers we pass to an algorithm to specify a tuple $p \in \mathcal{P}_{\boldsymbol{d}}[q]$.

An element $p \in \mathcal{P}_{\boldsymbol{d}}[q]$ determines $5 q$ atomic sets of the form $\left\{x \in \mathbb{R}^{n} \mid p_{i} \propto 0\right\}$, where $\propto \in\{<, \leq,=, \geq,>\}$ and $i \in[q]:=\{1, \ldots, q\}$. A Boolean combination (Boolean formula) of these sets is an expression recursively constructed from them by taking unions, intersections, and complements (respectively, disjunctions $\vee$, conjunctions $\wedge$, and negations $\neg)$. We will often refer to a Boolean formula over $p$ to mean a formula in the $5 q$ atomic relations above. For such a formula $\Phi$ we denote by size $(\Phi)$ the number of terms in its recursive construction. The quantity $\operatorname{size}(p, \Phi):=N+\operatorname{size}(\Phi)$ is therefore a measure of the size of a pair $(p, \Phi)$.

Any such pair defines a semialgebraic set $W(p, \Phi) \subseteq \mathbb{R}^{n}$ and we are interested here in computing, with input $(p, \Phi)$, the homology groups of $W(p, \Phi)$. We already observed that size $(p, \Phi)$ measures the input size for such an algorithm. Its cost on this input is the number of arithmetic operations and comparisons in $\mathbb{R}$ performed during the computation. In our algorithm, as in many numerical algorithms, this cost depends on size $(p, \Phi)$ but it is not bounded by a function of this quantity only. Instead, the cost (and the precision required to ensure a correct output when running the algorithm with finite precision) depends as well on a condition number $\bar{\kappa}_{\text {aff }}(p)$. This is a number in $[1, \infty]$. Tuples $p \in \mathcal{P}_{\boldsymbol{d}}[q]$ with $\bar{\kappa}_{\text {aff }}(p)=\infty$ are those for which, for some $\Phi$, arbitrarily small perturbations of the coefficients in $p$ may change the homology groups of $W(p, \Phi)$. Such tuples, called ill-posed, form a lower-dimensional semialgebraic subset of $\mathcal{P}_{\boldsymbol{d}}[q]$. We briefly recall some facts about $\bar{\kappa}_{\text {aff }}(p)$, and point to the relevant sections in [2] where these facts are shown, in 92.3 below.

To obtain weak complexity bounds we need to endow $\mathcal{P}_{\boldsymbol{d}}[q]$ with a probability measure and to do so, it will be convenient to have an inner product on this space. We endow $\mathcal{P}_{\boldsymbol{d}}[q]$ with the Weyl inner product (see $[2, \S 3.1]$ ) and the associated unit sphere $\mathbb{S}\left(\mathcal{P}_{\boldsymbol{d}}[q]\right)=\mathbb{S}^{N-1}$ with the uniform probability measure. With this measure at hand we can state our main result (whose general form is remarkably similar to the one of [2], the only difference being that the result here applies to arbitrary Boolean formulas).

Theorem 1.1. We exhibit a stable numerical algorithm Homology that, given a tuple $p \in \mathcal{P}_{\boldsymbol{d}}[q]$ and a Boolean formula $\Phi$ over $p$, computes the homology groups of $W(p, \Phi)$. The cost of Homology on input $(p, \Phi)$, denoted $\operatorname{cost}(p, \Phi)$, satisfies:

(i) $\operatorname{cost}(p, \Phi) \leq \operatorname{size}(\Phi) q^{\mathcal{O}(n)}\left(n D \bar{\kappa}_{\text {aff }}(p)\right)^{\mathcal{O}\left(n^{2}\right)}$.

Furthermore, if $p$ is drawn from the uniform distribution on $\mathbb{S}^{N-1}$, then:

(ii) $\operatorname{cost}(p, \Phi) \leq \operatorname{size}(\Phi) q^{\mathcal{O}(n)}(n D)^{\mathcal{O}\left(n^{3}\right)}$ with probability at least $1-(n q D)^{-n}$, and 
(iii) $\operatorname{cost}(p, \Phi) \leq 2^{\mathcal{O}\left(\operatorname{size}(p, \Phi)^{1+\frac{2}{D}}\right)}$ with probability at least $1-2^{-\operatorname{size}(p, \Phi)}$.

A few comments on Theorem 1.1;

(i) As in [2], we direct the reader to Section 7 of [6] for an explanation, along with a proof, of the numerical stability mentioned in the statement above. Details can also

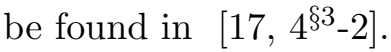

(ii) Part (iii) of Theorem 1.1 shows that Homology works in weak exponential time.

(iii) It is easy to check that all the routines in algorithm Homology do parallelize for the computation of the Betti numbers1. The parallel version of the algorithm can then be shown to work in parallel time $\operatorname{size}(p, \Phi)^{\mathcal{O}(1)}$ with probability at least $1-2^{-\operatorname{size}(p, \Phi)}$. That is, it works in weak parallel polynomial time. The arguments for this are in [2, $\S 7.4]$ (see also [17, $\left.4^{\S 3}-1\right]$ ).

(iv) We note that $\Phi$ can be rewritten as a formula in disjunctive normal form of size at most $(q D)^{\mathcal{O}(n)}$. Therefore one could in principle eliminate size $(\Phi)$ from the complexity estimates. However, we found it desirable to indicate the dependence of the cost of the algorithm in terms of all the intervening parameters, including the size of the formula.

Structure of the paper. In Section 2 we provide an overview of the various ingredients that make up our algorithms and its analysis. At the end of this section we are in a situation of describing the algorithm itself and give a proof of Theorem 1.1 based on the notions and results in this overview. Sections 3 and 4 provide the proofs of these results. Finally, we conclude in Section 5 with a discussion on how to combine numeric with symbolic algorithms.

\section{Contents}

1 Introduction

2 Overview of the Algorithm

2.1 Elimination of negations and lax inequalities . . . . . . . . . . . .

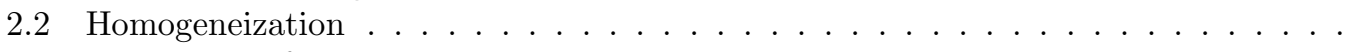

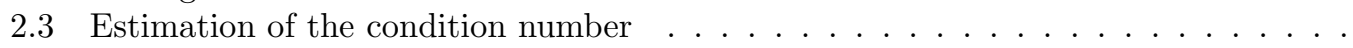

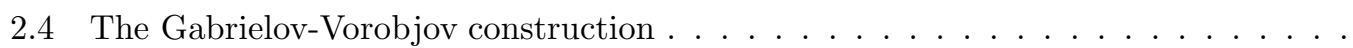

2.5 Reach and condition of Gabrielov-Vorobjov approximations . . . . . . . . . . . .

2.6 Point clouds and cell complexes . . . . . . . . . . . . . . . . . . . . 10

2.7 The algorithm . . . . . . . . . . . . . . . . . . . . . . . . . . . 11

3 Quantitative Gabrielov-Vorobjov Theorem $\quad 12$

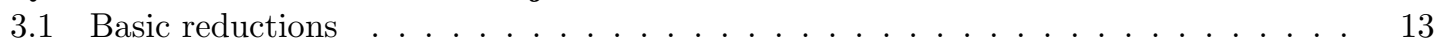

3.2 Some elements of differential topology . . . . . . . . . . . . . . . . . . . . . . . . . 15

3.2.1 Stratified sets and Mather-Thom theory . . . . . . . . . . . . 15

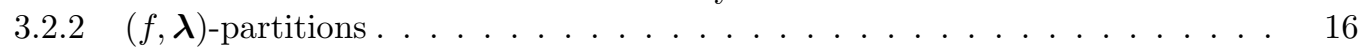

3.3 Proof of Proposition $3.2 \ldots \ldots \ldots \ldots \ldots \ldots \ldots \ldots$

3.3.1 Homotopies preserving $\Pi_{f, \boldsymbol{\lambda}} \ldots \ldots \ldots \ldots \ldots$

3.3.2 Proof of Proposition $3.11 \ldots \ldots \ldots \ldots \ldots$

\footnotetext{
${ }^{1}$ Claims in [1, 2] regarding the parallelization of the computation of torsion coefficients were inaccurate. As of today, the main difficulty lies in showing the existence of efficient parallel algorithms for the computation of the Smith Normal Form of integer matrices. See [17, p. 160-161] for more details.
} 
4 Sampling theory for Gabrielov-Vorobjov approximations $\quad 26$

4.1 Semialgebraic sets from $(f, t)$ and related results . . . . . . . . . . . . 26

4.2 Proof of Theorem $2.13 \ldots \ldots \ldots \ldots \ldots \ldots \ldots \ldots$. . . . . . . . . . . . . . . . . . . . . . . . . . . . . . . . . . . . . .

4.3 Proof of Theorem $2.14 \ldots \ldots \ldots \ldots \ldots \ldots$

4.4 Proof of Theorem 2.15 . . . . . . . . . . . . . . . . . . . . . 31

5 Concluding remarks $\quad 31$

Acknowledgments. We are grateful to Nicolai Vorobjov who pointed us to (what we call here) Gabrielov-Vorobjov approximations.

\section{Overview of the Algorithm}

\subsection{Elimination of negations and lax inequalities}

The initial step in our algorithm eliminates negations and lax inequalities (in this order) in the given formula $\Phi$. This can be done in time linear in size $(\Phi)$ and the resulting formula after this elimination has a size which is at most $2 \operatorname{size}(\Phi)$, the increase being due to the substitutions

$$
p \geq 0 \rightsquigarrow(p=0 \vee p>0)
$$

and

$$
p \leq 0 \rightsquigarrow(p=0 \vee p<0) .
$$

All along this paper, we will write $\alpha \rightsquigarrow \beta$ to indicate that an expression $\alpha$ is rewritten as (i.e., replaced by) another expression $\beta$.

The resulting formula is therefore monotone (no negations) and has no lax inequalities: it is built over the $3 q$ atoms $p_{i} \propto 0$ with $i \in[q]$ and $\propto \in\{<,=,>\}$. We will call these formulas strict.

Strict formulas can be rewriten in Disjunctive Normal Form (DNF) and the resulting formula is also strict (as atoms remain unchanged). Even though we will not need to convert the input formula into DNF in our algorithm, a conversion that may exponentially increase its size, we will use DNFs in many of our reasonings. We therefore recall that we call purely conjunctive a conjunction of atoms and that semialgebraic sets given by purely conjunctive formulas are called basic.

\subsection{Homogeneization}

From now on, we assume that $\Phi$ is strict. The next step in our algorithm maps into the sphere the semialgebraic set $W(p, \Phi)$ by considering its spherical closure. To do so, we recall some notation.

As before, let $\boldsymbol{d}=\left(d_{1}, \ldots, d_{q}\right)$ be a $q$-tuple of positive integers. We denote by $\mathcal{H}_{\boldsymbol{d}}[q]$ the vector space of $q$-tuples $f=\left(f_{1}, \ldots, f_{q}\right)$ of homogeneous polynomials $f_{i} \in \mathbb{R}\left[X_{0}, \ldots, X_{n}\right]$ of degree $d_{i}$. We put $\boldsymbol{d}^{*}:=(1, \boldsymbol{d})$. The homogenization map $\mathbf{H}: \mathcal{P}_{\boldsymbol{d}}[q] \rightarrow \mathcal{H}_{\boldsymbol{d}^{*}}[q+1]$ is defined by

$$
p \mapsto \mathrm{H}(p):=\left(\|p\| X_{0}, p_{1}^{\mathrm{h}}, \ldots, p_{q}^{\mathrm{h}}\right),
$$

where $p_{i}^{\mathrm{h}}:=p_{i}\left(X_{1} / X_{0}, \ldots, X_{n} / X_{0}\right) X_{0}^{d_{i}}$ denotes the homogenization of $p_{i}$ and $\|p\|$ stands for the Weyl norm of the tuple $p$ (see [2, §3.1]).

Any formula $\Phi$ over $f \in \mathcal{H}_{\boldsymbol{d}}[q]$ defines a semialgebraic subset $\mathrm{S}(f, \Phi)$ of the sphere $\mathbb{S}^{n}$. It will be convenient to call these sets spherical semialgebraic. In order to simplify the notation, we will also write $\mathrm{S}(f=0)$ etc. with the obvious meaning. 
The following result is straightforward.

Proposition 2.1. Let $p \in \mathcal{P}_{\boldsymbol{d}}[q]$ and $\Phi$ be a strict formula over $p$. Denote by $\Phi^{\mathrm{h}}$ the formula over $\mathrm{H}(p) \in \mathcal{H}_{\boldsymbol{d}^{*}}[q+1]$ given by

$$
\Phi^{\mathrm{h}}:=\Phi\left(p_{1}^{\mathrm{h}}, \ldots, p_{q}^{\mathrm{h}}\right) \wedge\left(\|p\| X_{0}>0\right) .
$$

Then the sets $W(p, \Phi)$ and $\left.\mathbf{S}\left(\mathbf{H}(p), \Phi^{\mathrm{h}}\right)\right)$ are homeomorphic.

\subsection{Estimation of the condition number}

In $[2, \S 3.4]$ we defined a condition number $\bar{\kappa}(f) \in[1, \infty]$ associated to a tuple $f \in \mathcal{H}_{\boldsymbol{d}}[q]$, whose inverse measures how near are the intersections between the hypersurfaces given by $f$ from being non-transversal. The condition number $\bar{\kappa}_{\text {aff }}(p)$ in Theorem 1.1 was then defined [2, §7.1] to be $\bar{\kappa}(\mathrm{H}(p)))$. The quantity $\bar{\kappa}(f)$ provides information on the geometry of every possible spherical semialgebraic set built from $f$. Tuples $f$ for which $\bar{\kappa}(f)=\infty$ are said to be ill-posed. They are precisely those tuples for which there exists a formula $\Phi$ such that arbitrary small perturbations of $f$ may change the topology of $\mathbf{S}(f, \Phi)$. The set $\bar{\Sigma}_{\boldsymbol{d}}[q]$ of ill-posed tuples has positive codimension in $\mathcal{H}_{\boldsymbol{d}}[q]$ and $\bar{\kappa}(f)$ estimates how far is $f$ from $\bar{\Sigma}_{\boldsymbol{d}}[q]$.

The first substantial computational effort performed by Homology is to estimate the condition number $\bar{\kappa}(f)$ of a tuple $f \in \mathcal{H}_{\boldsymbol{d}}[q]$. The following result, Proposition 2.2 in [2], deals with this task.

Proposition 2.2. There is an algorithm $\bar{\kappa}$-Estimate that, given $f \in \mathcal{H}_{\boldsymbol{d}}[q]$, returns a number $\mathrm{K}$ such that

$$
0.99 \bar{\kappa}(f) \leq \mathrm{K} \leq \bar{\kappa}(f)
$$

if $\bar{\kappa}(f)<\infty$, or loops forever otherwise. The cost of this algorithm is bounded by $(q n D \bar{\kappa}(f))^{\mathcal{O}(n)}$.

When $\bar{\kappa}(f)$ is infinity the algorithm loops forever because it cannot find an upper bound for $\bar{\kappa}(f)$. However, we note that the algorithm in [2] can be modified to stop if $\bar{\kappa}(f)$ is too large and return this fact (see [2, Proposition 6.3]).

\subsection{The Gabrielov-Vorobjov construction}

The main idea behind the algorithm in 2] consists of finding a finite collection of points $\mathcal{X}$ and a radius $\varepsilon$ such that the union $\cup_{x \in \mathcal{X}} B(x, \varepsilon)$ contains the set $\mathrm{S}(f, \Phi)$ and continuously retracts to it. Out of the realm of lax formulas we dealt with in [2] this idea becomes impracticable. The reason is that the connected components of $\mathrm{S}(f, \Phi)$ may now not be separated by a positive distance. Consider for instance the pair $f=(X-Y, Y)$ and the semialgebraic set given by

$$
\Phi \equiv(X-Y=0 \wedge Y>0) \vee(Y=0 \wedge X-Y>0) .
$$

The set $\mathrm{S}(f, \Phi)$ consists of two open half-lines with origin at $(0,0)$. Balls close to $(0,0)$ containing initial segments of the two half-lines are likely to intersect.

To circumvent this problem we will rely on a beautiful construction conceived by A. Gabrielov and N. Vorobjov in [8] that produces closed semialgebraic approximations to semialgebraic sets. These are obtained by combining relaxations of the equalities and strengthenings of the inequalities in the formula $\Phi$. These relaxations and strengthenings can be seen as a rewriting of the formula $\Phi(f)$ into a new formula. 
Definition 2.3. Given a monotone formula $\Phi$ over $f \in \mathcal{H}_{\boldsymbol{d}}[q]$ and positive $\delta$ and $\varepsilon$, the Gabrielov-Vorobjov $(\delta, \varepsilon)$-block $\Gamma \mathrm{B}_{\delta, \varepsilon}(f, \Phi)$ is the spherical semialgebraic set defined by the following rewriting of $\Phi(f)$,

$$
\begin{aligned}
& f_{i}=0 \rightsquigarrow\left|f_{i}(x)\right| \leq \varepsilon\left\|f_{i}\right\|, \\
& f_{i}>0 \rightsquigarrow f_{i}(x) \geq \delta\left\|f_{i}\right\|, \text { and } \\
& f_{i}<0 \rightsquigarrow f_{i}(x) \leq-\delta\left\|f_{i}\right\| .
\end{aligned}
$$

Given $\boldsymbol{\delta}, \boldsymbol{\varepsilon} \in(0, \infty)^{m}$, the Gabrielov-Vorobjov $(\boldsymbol{\delta}, \boldsymbol{\varepsilon})$-approximation $\Gamma_{\boldsymbol{\delta}, \boldsymbol{\varepsilon}}(f, \Phi)$ (of or$\operatorname{der} m)$ of $\mathbf{S}(f, \Phi)$ is the spherical semialgebraic set given by

$$
\Gamma \mathrm{B}_{\boldsymbol{\delta}, \boldsymbol{\varepsilon}}(f, \Phi):=\bigcup_{k=1}^{m} \Gamma \mathrm{B}_{\delta_{k}, \varepsilon_{k}}(f, \Phi) .
$$

Note that Gabrielov-Vorobjov blocks and Gabrielov-Vorobjov approximations are compact subsets of $\mathbb{S}^{n}$. The norms $\left\|f_{i}\right\|$ in the definition above are not in [8]. We have added them here as they make clearer statements in our context.

The main result of [8], Theorem 1.10 there, yields the following immediate consequence (which also holds with our modified definition of Gabrielov-Vorobjov blocks). Recall that $\pi_{k}$ stands for the $k$ th homotopy group and $H_{k}$ for the $k$ th homology group.

Theorem 2.4 (Gabrielov-Vorobjov Theorem). Let $f \in \mathcal{H}_{\boldsymbol{d}}[q]$, $\Phi$ be a monotone formula over $f, m \in \mathbb{N}$, and $\boldsymbol{\delta}, \boldsymbol{\varepsilon} \in(0, \infty)^{m}$. If

$$
0<\varepsilon_{1} \ll \delta_{1} \ll \cdots \ll \varepsilon_{m} \ll \delta_{m} \ll 1,
$$

then, for $k \in\{0, \ldots, m-1\}$, there are homomorphisms

$$
\phi_{k}: \pi_{k}\left(\Gamma \mathrm{B}_{\delta, \varepsilon}(f, \Phi)\right) \rightarrow \pi_{k}(\mathrm{~S}(f, \Phi))
$$

and

$$
\varphi_{k}: H_{k}\left(\Gamma_{\delta, \varepsilon}(f, \Phi)\right) \rightarrow H_{k}(\mathrm{~S}(f, \Phi))
$$

that are isomorphisms for $k<m-1$ and epimorphisms when $k=m-1$.

In this statement, the relations $0<a_{1} \ll \cdots \ll a_{t} \ll 1$ of reals $a_{i}$ mean that there are functions $h_{k}:(0,1)^{t-k} \rightarrow(0,1)$ such that $0<a_{k}<h_{k}\left(a_{k+1}, \ldots, a_{t}\right)$ for all $k$.

Remark 2.5. Homotopy groups (without specifying a base point) are only defined for connected spaces. However, the bijection between $\pi_{0}\left(\Gamma_{\delta, \varepsilon}(f, \Phi)\right)$ and $\pi_{0}(\mathrm{~S}(f, \Phi))$ identifies the connected components of $\Gamma_{B_{\delta, \varepsilon}}(f, \Phi)$ and $\mathrm{S}(f, \Phi)$. Therefore we can naturally interpret $\phi_{k}: \pi_{k}\left(\Gamma_{\delta, \varepsilon}(f, \Phi)\right) \rightarrow \pi_{k}(\mathrm{~S}(f, \Phi))$, for $k>0$, as the family of maps

$$
\left\{\phi_{k}: \pi_{k}(C) \rightarrow \pi_{k}\left(\phi_{0}(C)\right) \mid C \in \pi_{0}\left(\Gamma_{\delta, \varepsilon}(f, \Phi)\right)\right\} .
$$

The assumption of connectedness in [8] is only for technical ease of the exposition.

The proof of Theorem 2.4 is an elegant conjunction of geometric insight and technical skill. While it is out of our reach to explain the ideas behind it (the interested reader will find these ideas in [8]) we believe a few simple examples may provide some intuition. 


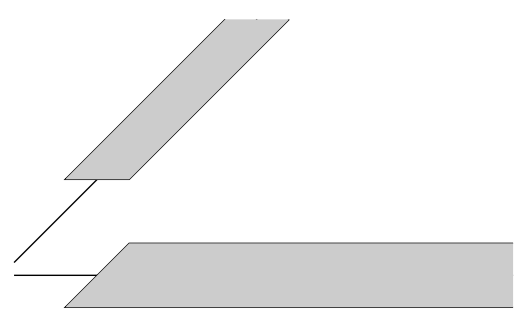

Figure 1 The Gabrielov-Vorobjov construction for two open half-lines

Example 2.6. Consider the pair $f$ and formula $\Phi$ in (2.1) we started this subsection with. For any pair $(\delta, \varepsilon)$ with $0<\varepsilon<\delta$ the block $\Gamma_{\delta, \varepsilon}(f, \Phi)$ is given by

$$
(|X-Y| \leq \varepsilon \sqrt{2} \wedge(Y \geq \delta)) \vee(|Y| \leq \varepsilon \wedge(X-Y \geq-\delta \sqrt{2}))
$$

and looks as in Figure 1.

It is clear that this block is homotopically equivalent to $\mathrm{S}(f, \Phi)$.

Example 2.7. The number $m$ of blocks needed in the Gabrielov-Vorobjov construction to recover the $k$ th homology group of $\mathrm{S}(f, \Phi)$ may reach the bound $k+2$ in Theorem 2.4 . Let $f=(X, Y)$ and consider

$$
\Phi \equiv(X=0 \wedge Y=0) \vee(X=0 \wedge Y>0) \vee(X>0 \wedge Y=0) \vee(X>0 \wedge Y>0)
$$

so that $\mathrm{S}(f, \Phi)$ is the closed positive quadrant. Now take any sequence

$$
0<\varepsilon_{1}<\delta_{1}<\varepsilon_{2}<\delta_{2}<\varepsilon_{3}<\delta_{3} .
$$

At the left of Figure 2 we see in light grey shading the block $\Gamma_{\delta_{1}, \varepsilon_{1}}(f, \Phi)$. It is not connected; not even the 0th homology group is correct. At the center of the figure we see that same first block with $\Gamma \mathrm{B}_{\delta_{2}, \varepsilon_{2}}(f, \Phi)$ superimposed in a darker shade of grey. Now the union of the first two blocks is connected (so $H_{0}$ is correct) but not simply connected: the first homology group is wrong. We obtain a contractible set, homotopically equivalent to $\mathrm{S}(f, \Phi)$, when we add the third block, at the right of the figure, to the union.

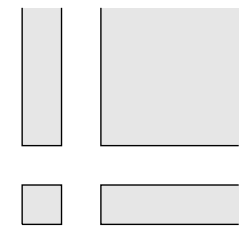

First $\Gamma \mathrm{B}$ block

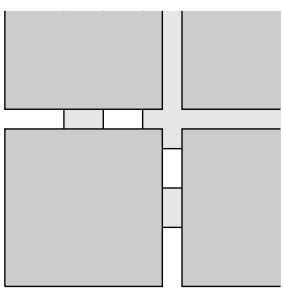

First two ГВ blocks

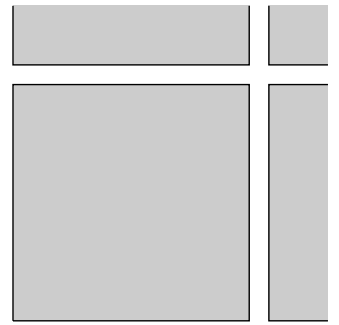

Third ГВ block

Figure 2 The Gabrielov-Vorobjov construction for the positive quadrant

We now remark that no explicit form of the functions $h_{k}$ behind the relations $0<$ $a_{1} \ll \cdots \ll a_{t} \ll 1$ is given in [8]. Our first main result, Theorem 2.8 below, provides a very simple answer to this issue for well-posed tuples of polynomials. 
Theorem 2.8 (Quantitative Gabrielov-Vorobjov Theorem). In Theorem 2.4, condition (2.3) can be replaced by

$$
0<\varepsilon_{1}<\delta_{1}<\cdots<\varepsilon_{m}<\delta_{m}<\frac{1}{\sqrt{2} \bar{\kappa}(f)}
$$

when $\bar{\kappa}(f)<\infty$.

Example 2.9. The simple form of the inequalities in (2.4) requires well-posedness, i.e., $\bar{\kappa}(f)<\infty$. To see this, consider $f=(X, Y, X-Y)$ and

$$
\Phi \equiv((X=0) \wedge(Y>0)) \vee((X-Y=0) \wedge(Y>0))
$$

The set $\mathrm{S}(f, \Phi)$ consists of two half-lines with a common origin but without this origin. Note that $\bar{\kappa}(f)=\infty$. Figure 3 shows $\mathbf{S}(f, \Phi)$ at the left. The center and right parts of the

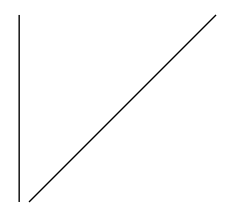

$\mathrm{S}(f, \Phi)$

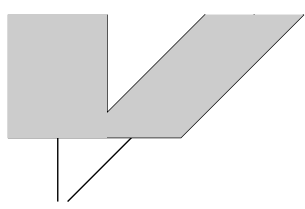

$\Gamma_{0.75,0.5}(f, \Phi)$

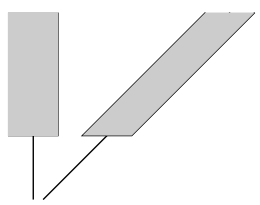

$\Gamma_{0.75,0.25}(f, \Phi)$

Figure 3 The Gabrielov-Vorobjov construction for an ill-posed system

figure exhibit two Gabrielov-Vorobjov Approximations for it with $m=1$ but different pairs $(\delta, \varepsilon)$. The middle part shows that the condition $\varepsilon<\delta$ is not strong enough to guarantee the conclusions of Theorem 2.4 for $m=1$. An easy computation shows that, in this case, we need $0<\varepsilon<\delta / 2$ (as in the right part of the figure).

\subsection{Reach and condition of Gabrielov-Vorobjov approximations}

In the following let $f \in \mathcal{H}_{\boldsymbol{d}}[q]$ be such that $\bar{\kappa}(f)<\infty$. Moreover let $\Phi$ be a strict formula over $f$ and the integer $m$ satisfy $m \geq 2+\operatorname{dim} \mathrm{S}(f, \Phi)$; such $m$ can be easily computed since $f$ is well-posed. Moreover, let $\mathrm{K}$ be an estimate of $\bar{\kappa}(f)$ as in Proposition 2.2. Using the increasing tuple of positive reals

$$
\left(\varepsilon_{1}, \delta_{1}, \ldots, \varepsilon_{m}, \delta_{m}\right):=\left(15(2 m+1) D^{2} \mathrm{~K}^{2}\right)^{-1}(1,2, \ldots, 2 m),
$$

we define the Gabrielov-Vorobjov approximation of $\mathrm{S}(f, \Phi)$

$$
\Gamma \mathrm{B}(f, \Phi):=\Gamma_{\boldsymbol{\delta}, \boldsymbol{\varepsilon}}(f, \Phi) .
$$

By Theorem 2.8, $\Gamma \mathrm{B}(f, \Phi)$ and $\mathrm{S}(f, \Phi)$ have the same homology.

As $\Gamma \mathrm{B}(f, \Phi)$ is closed, a first idea would be to apply to it the algorithm developed in [2]. Unfortunately, as we are about to see, this idea does not work. Let $e:=4 m$ and abbreviate

$$
t:=\left(t_{1}, \ldots, t_{e}\right):=\left(\varepsilon_{1}, \delta_{1}, \ldots, \varepsilon_{m}, \delta_{m},-\varepsilon_{1},-\delta_{1}, \ldots,-\varepsilon_{m},-\delta_{m}\right),
$$


where the $\varepsilon_{j}, \delta_{j}$ are those in (2.5). We note that $\Gamma \mathrm{B}(f, \Phi)$ is defined by a Boolean formula $\bar{\Phi}$ (depending on $\Phi$ ) in terms of the tuple of polynomials

$$
\bar{f}:=\left\{f_{i}-t_{j}\left\|f_{i}\right\|\right\}_{i \leq q, j \leq e} .
$$

But the polynomials in $\bar{f}$ are no longer homogeneous. It is easy to verify that, in general, $\bar{\kappa}_{\text {aff }}(\bar{f})=\infty$, since the $f_{i}-t_{j}\left\|f_{i}\right\|$, for different $j$, intersect tangentially at infinity whenever $f_{i}$ is non-linear. Thus it is hopeless to pass $\bar{f}$ as input to the algorithm described and analyzed in [2].

A closer look to this analysis reveals however, that its main ideas can be reproduced in our situation. The first stepping stone in [2] towards the algorithm's design is the following result (Theorem 2.3 there).

Theorem 2.10 (Basic Homotopy Witness Theorem I). Let $f \in \mathcal{H}_{\boldsymbol{d}}[q]$ and $\phi$ be a purely conjunctive lax formula over $f$. Moreover, let $\mathcal{X} \subseteq \mathbb{S}^{n}$ be a closed subset and $\varepsilon>0$ be such that

$$
3 d_{H}(\mathcal{X}, \mathrm{S}(f, \phi))<\varepsilon<\frac{1}{14 D^{\frac{3}{2}} \bar{\kappa}(f)} .
$$

Then the inclusion $\mathrm{S}(f, \phi) \hookrightarrow \mathcal{U}(\mathcal{X}, \varepsilon)$ induces a homotopy equivalence.

In this statement, $d_{H}$ denotes the Hausdorff distance between two nonempty compact sets $W, V \subseteq \mathbb{R}^{n+1}$ which, we recall, is given by

$$
d_{H}(W, V):=\max \left\{\max _{v \in V} d(W, v), \max _{w \in W} d(w, V)\right\}
$$

where $d$ denotes Euclidean distance in $\mathbb{R}^{n+1}$. If either $V$ or $W$ is empty then one takes $d_{H}(V, W):=\infty$, unless both are empty, in which case $d_{H}(V, W):=0$. Also,

$$
\mathcal{U}(\mathcal{X}, r):=\bigcup_{x \in \mathcal{X}} B(x, r)
$$

denotes the open $r$-neighborhood of $\mathcal{X}$ in $\mathbb{R}^{n+1}$ and $B(x, r)$ the Euclidean open ball with center $x$ and radius $r$.

Theorem 2.10 goes back to a result by Niyogi, Smale and Weinberger [12] proving the statement for manifolds and expressing the bound on the right-hand side in terms of the reach of the manifold. For submanifolds $\mathcal{M}$ of $\mathbb{S}^{n}$ given as the zero set of a tuple $f$ of homogeneous polynomials, this reach was bounded in [6] in terms of $\max _{x \in \mathcal{M}} \gamma(f, x)$ where $\gamma(f, x)$ is the $\gamma$-invariant introduced by Smale [16]. This result was given a much simpler proof in [1, Theorem 2.11]. The Higher Derivative Estimate [15, 3] further allowed one to replace $\gamma$ by the condition number.

In Section 4 we will take advantage of this sequence of results. Basically, we will show that the $\gamma$ at points of algebraic sets defined using polynomials from $\bar{f}$ can be bounded in terms of the $\gamma$ for $f$ instead and, hence, ultimately in terms of $\bar{\kappa}(f)$. We will then derive an extension of Theorem 2.10 appropriate to our setting.

It is convenient to introduce some more terminology.

Definition 2.11. Let $f \in \mathcal{H}_{\boldsymbol{d}}[q]$ and $t \in \mathbb{R}^{e}$. We call a lax formula $\Phi$ over $(f, t)$ a monotone formula, whose atoms are of the form $\left(f_{i} \geq t_{j}\left\|f_{i}\right\|\right)$ or $\left(f_{i} \leq t_{j}\left\|f_{i}\right\|\right)$, with $i \leq q$ and $j \leq e$ and denote by $\mathrm{S}(f, t, \Phi)$ the semialgebraic subset of the sphere $\mathbb{S}^{n}$ described by these lax inequalities. 
Remark 2.12. The set $\mathrm{S}(f, t, \Phi)$ is defined in terms of sign conditions on the polynomials in $\bar{f}$ (see (2.8)), which are not homogeneous. Every intersection of Gabrielov-Vorobjov blocks $\Gamma \mathrm{B}_{\delta_{j}, \varepsilon_{j}}(f, \phi)$ is of the form $\mathrm{S}(f, t, \phi)$ for a purely conjunctive formula $\phi$. Moreover, the formula $\bar{\Phi}$ defined right before (2.8) can be viewed as a formula over $(f, t)$ such that $\Gamma \mathrm{B}(f, \Phi)=\mathrm{S}(f, t, \bar{\Phi})$, by the construction of the Gabrielov-Vorobjov approximations.

Theorem 2.13 (Generalized Basic Homotopy Theorem). Let $f \in \mathcal{H}_{\boldsymbol{d}}[q], T>0$ be such that $\sqrt{2} \bar{\kappa}(f) T<1, t \in(-T, T)^{e}$, and $\phi$ a purely conjunctive lax formula over $(f, t)$. Moreover, let $\mathcal{X} \subseteq \mathbb{S}^{n}$ be a closed subset and $\varepsilon>0$ be such that

$$
3 d_{H}(\mathcal{X}, \mathrm{S}(f, t, \phi))<\varepsilon<\frac{1}{48 D^{\frac{3}{2}} \bar{\kappa}(f)} .
$$

Then the inclusion $\mathrm{S}(f, t, \phi) \hookrightarrow \mathcal{U}(\mathcal{X}, \varepsilon)$ is a homotopy equivalence.

We prove this result in 94.2 ,

\subsection{Point clouds and cell complexes}

For a finite set $\mathcal{X} \subseteq \mathbb{R}^{n+1}$, we compute the homology groups of $\mathcal{U}(\mathcal{X}, \varepsilon)$ by computing those of the $\check{C}$ ech complex $\check{\mathrm{C}}_{\varepsilon}(\mathcal{X})$ which is the simplicial complex whose $k$-faces are the sets of $k+1$ points $\left\{x_{0}, \ldots, x_{k}\right\} \subseteq \mathcal{X}$ such that $\cap_{i \leq k} B\left(x_{i}, \varepsilon\right) \neq \varnothing$. The justification of this is the Nerve Theorem [10, Corollary 4G.3] that guarantees that these two spaces are homotopically equivalent.

Theorem 2.13 opens the way to find a good pair $(\mathcal{X}, \varepsilon)$ for basic sets. For non-basic sets, one needs a variation, where the simplicial complex is constructed in a more complicated way. We recall this construction. For given $f \in \mathcal{H}_{\boldsymbol{d}}[q]$ and $t \in \mathbb{R}^{e}$ consider the $2 q$ e atomic sets

$$
\begin{aligned}
& S_{i, j}^{\leq}:=\mathrm{S}\left(f_{i}-t_{j}\left\|f_{i}\right\| \leq 0\right), \\
& S_{i, j}^{\geq}:=\mathrm{S}\left(f_{i}-t_{j}\left\|f_{i}\right\| \geq 0\right),
\end{aligned}
$$

for $i \leq q$ and $j \leq e$. Furthermore, also for $i \leq q$ and $j \leq e$, let $\mathcal{X}_{i, j}^{\leq}$and $\mathcal{X}_{i, j}^{\geq}$be finite subsets of $\mathbb{S}^{n}$. Given a lax formula $\Psi$ over $(f, t)$, we can abuse notation and write $\Phi\left(\mathcal{X}_{i, j}^{\propto}\right)$ to denote the finite set recursively built from the sets $\mathcal{X}_{i, j}^{\propto}$ in the same way $\Psi$ is built from the expressions $\left(f_{i} \propto t_{j}\left\|f_{i}\right\|\right)$, taking unions when we find $\vee$ and intersections when we find $\wedge$. As unions and intersections of the simplicial complexes $\check{C}_{\varepsilon}\left(\mathcal{X}_{i, j}^{\propto_{j}}\right)$ are well defined, we can further abuse notation to denote by

$$
\Psi\left(\check{\mathrm{C}}_{\varepsilon}\left(\mathcal{X}_{i, j}^{\propto}\right) \mid i \in[q], j \in[e], \propto \in\{\leq, \geq\}\right) \subseteq \Delta^{\cup_{i, j, \propto} \mathcal{X}_{i, j}^{\propto}},
$$

the simplicial complex recursively built from the $\check{\mathrm{C}}_{\varepsilon}\left(\mathcal{X}_{i, j}^{\propto_{j}}\right)$. This is explained with more details in [2, §2.4]. Applying $\Psi$ to a collection of point clouds or to one of simplicial complexes is an abuse of notation but it allows us to clearly state the following extension of Theorem 2.13,

Theorem 2.14 (Generalized Homology Witness Theorem). Let $f \in \mathcal{H}_{\boldsymbol{d}}[q]$, $\mathrm{K}$ be as in Proposition [2.2, and $t \in \mathbb{R}^{4 m}$ as in (2.7). Let $\varepsilon>0$, and assume that for $i \in[q]$ 
and $j \in[4 m], \mathcal{X}_{i, j}^{\leq}$and $\mathcal{X}_{i, j}^{\geq}$are finite subsets of $\mathbb{S}^{n}$ such that for all purely conjunctive lax formulas $\phi$ over $(f, t)$, we have

$$
3 d_{H}\left(\phi\left(\mathcal{X}_{i, j}^{\propto} \mid i \in[q], j \in[4 m], \propto \in\{\leq, \geq\}\right), \mathrm{S}(f, t, \phi)\right)<\varepsilon<\frac{1}{180(2 m+1) D^{5 / 2} \mathrm{~K}^{2}} .
$$

Then, for all strict formulas $\Phi$ over $f$, the Gabrielov-Vorobjov approximation $\Gamma \mathrm{B}(f, \Phi)$ defined in (2.6) and the simplicial complex

$$
\mathfrak{C}=\bar{\Phi}\left(\check{\mathrm{C}}_{\varepsilon}\left(\mathcal{X}_{i, j}^{\propto}\right) \mid i \in[q], j \in[4 m], \propto \in\{\leq, \geq\}\right)
$$

have the same homology.

We prove this result in 4.3 .

To make use of Theorem 2.14 we need to construct finite sets $\mathcal{X}_{i, j}^{\leq}$and $\mathcal{X}_{i, j}^{\geq}$satisfying (2.11). As in [2], to do this, we will rely on the notion of an algebraic neighborhood (see 4 4.1), now adapted to our context, and on the use of grids.

For $\ell \in \mathbb{N}$ one can construct a grid $\mathcal{G}_{\ell} \subseteq \mathbb{S}^{n}$ such that

$$
\left|\mathcal{G}_{\ell}\right| \leq\left(n 2^{\ell}\right)^{\mathcal{O}(n)} \quad \text { and } \quad \mathbb{S}^{n} \subseteq \cup_{x \in \mathcal{G}_{\ell}} B_{\mathbb{S}}\left(x, r_{\ell}\right) \subset \cup_{x \in \mathcal{G}_{\ell}} B\left(x, r_{\ell}\right),
$$

where $r_{\ell}:=2^{-\ell}$ (see $\left.[2, \S 6.1]\right)$ and $B_{\mathbb{S}}\left(x, r_{\ell}\right)$ is the ball centered at $x$ of radius $r_{\ell}$ with respect to the Riemannian distance on the sphere.

Given $f \in \mathcal{H}_{\boldsymbol{d}}[q], t \in \mathbb{R}^{e}$, for each $i \in[q], j \in[e]$, we consider the set $\mathcal{X}_{i, j}^{\geq}$of grid points $x$ in $\mathcal{G}_{\ell}$ satisfying

$$
f_{i}(x)>\left(t_{j}+D^{\frac{1}{2}} r_{\ell}\right)\left\|f_{i}\right\|,
$$

which means that the inequality $f_{i}>t_{j}\left\|f_{i}\right\|$ holds true with the tolerance $D^{\frac{1}{2}} r_{\ell}$. Similarly, we define the set of of grid points $\mathcal{X}_{i, j}^{\leq}$.

In 4.4 we prove the following result (generalizing Theorem 6.5 in [1]).

Theorem 2.15 (Generalized Sampling Theorem). Let $f \in \mathcal{H}_{\boldsymbol{d}}[q]$, K be as in Proposition 2.2, and $t \in \mathbb{R}^{4 m}$ as in (2.7). Moreover, let $\ell \in \mathbb{N}$ and $\rho \in(0,1]$ be chosen such that $r_{\ell} \leq \frac{\rho}{1620(2 m+1) D^{3} \mathrm{~K}^{3}}$. Then, for all purely conjunctive lax formulas $\phi$ over $(f, t)$, we have

$$
3 d_{H}\left(\phi\left(\mathcal{X}_{i, j}^{\propto} \mid i \in[q], j \in[4 m], \propto \in\{\leq, \geq\}\right), \mathrm{S}(f, t, \phi)\right) \leq \frac{\rho}{180(2 m+1) D^{5 / 2} \mathrm{~K}^{2}} .
$$

\subsection{The algorithm}

We can finally combine all the previous ideas in an algorithm and prove our main result. 


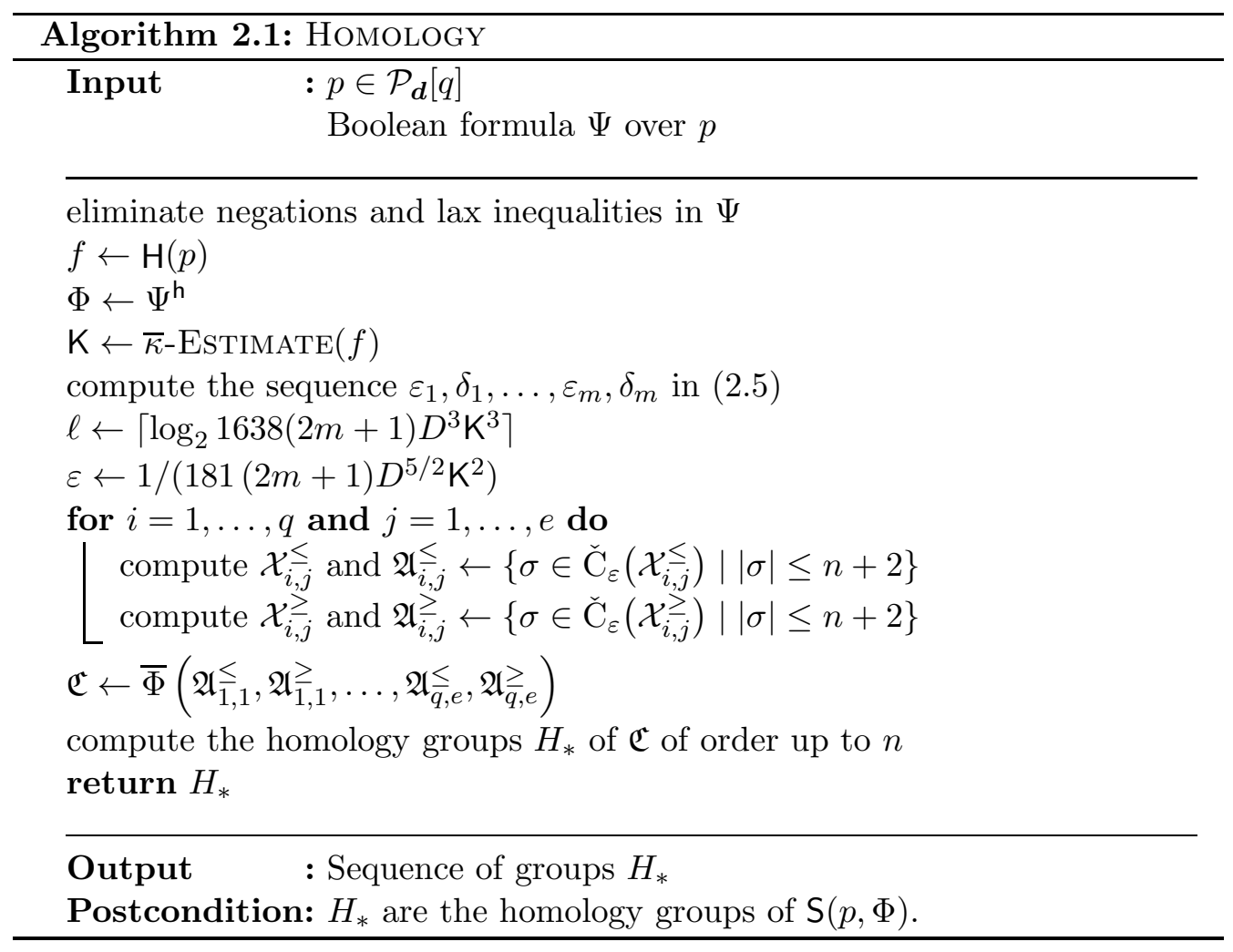

Proof of Theorem 1.1. The computation of $H_{*}$ from $\mathfrak{C}$ is described with details in 6 , Proposition 4.3].

As for the correctness of the algorithm. The choice of $\ell$ guarantees that

$$
r_{\ell}=2^{-\ell} \leq \frac{1}{1638(2 m+1) D^{3} \mathrm{~K}^{3}}=\frac{1620 / 1638}{1620(2 m+1) D^{3} \mathrm{~K}^{3}} .
$$

Thus Theorem 2.15 applies with $\rho=1620 / 1638$ and gives the upper bound $\frac{1}{182(2 m+1) D^{5 / 2} \mathrm{~K}^{2}}$. By Theorem 2.14, it is enough then to choose $\varepsilon$ inside

$$
\left(\frac{1}{182(2 m+1) D^{5 / 2} \mathrm{~K}^{2}}, \frac{1}{180(2 m+1) D^{5 / 2} \mathrm{~K}^{2}}\right) .
$$

Our choice of $\varepsilon$ satisfies these bounds. Hence, we have the homology isomorphisms

$$
H_{*}(\mathfrak{C}) \simeq H_{*}(\Gamma \mathrm{B}(f, \Phi)) \simeq H_{*}(\mathrm{~S}(f, \Phi)) \simeq H_{*}(W(p, \Psi)),
$$

the first by Theorem 2.14, the second by Theorem 2.8 and our choice of $\varepsilon_{1}, \delta_{1}, \ldots, \varepsilon_{m}, \delta_{m}$, and the last by Proposition 2.1, We note that we only care about the homology groups up to order $n$, since higher homology groups of a semialgebraic set in $\mathbb{R}^{n}$ vanish.

Finally, the cost analysis is the same as that in [2, $\S 7.3]$.

\section{Quantitative Gabrielov-Vorobjov Theorem}

The objective of this section is to prove the Quantitative Gabrielov-Vorobjov Theorem 2.8. 
The idea of the proof is simple, we transform a pair $(\boldsymbol{\delta}, \boldsymbol{\varepsilon})$ satisfying (2.4) into a pair $\left(\boldsymbol{\delta}^{\prime}, \varepsilon^{\prime}\right)$ satisfying (2.3) so that Theorem 2.4 can be applied and we show that in doing so, the homotopy type of the associated Gabrielov-Vorobjov set remains unchanged. To simplify the argument, we proceed by steps, modifying only one component in the pair $(\boldsymbol{\delta}, \boldsymbol{\varepsilon})$ at a time. In the next subsection we describe these basic reductions, leading us to a statement, Proposition 3.2, which implies Theorem 2.8, Then, in $\$ 3.2$ we recall some fundamental notions from differential topology which we use in $\$ 3.3$ to prove Proposition 3.2 .

\subsection{Basic reductions}

We write $(\boldsymbol{\delta}, \boldsymbol{\varepsilon}) \leq_{\mathcal{D}, i}\left(\boldsymbol{\delta}^{\prime}, \boldsymbol{\varepsilon}^{\prime}\right)$ when $\boldsymbol{\varepsilon}=\boldsymbol{\varepsilon}^{\prime}, \delta_{j}=\delta_{j}^{\prime}$ for $j \neq i$, and $\delta_{i} \geq \delta_{i}^{\prime}$. Similarly, we write $(\boldsymbol{\delta}, \boldsymbol{\varepsilon}) \leq_{\mathcal{E}, i}\left(\boldsymbol{\delta}^{\prime}, \boldsymbol{\varepsilon}^{\prime}\right)$ when $\boldsymbol{\delta}=\boldsymbol{\delta}^{\prime}, \varepsilon_{j}=\varepsilon_{j}^{\prime}$ for $j \neq i$, and $\varepsilon_{i} \leq \varepsilon_{i}^{\prime}$. Note that the calligraphic index $\mathcal{D}$ indicates a difference in a $\delta$ (and therefore, in an inequality of the corresponding Gabrielov-Vorobjov system), while a calligraphic $\mathcal{E}$ does so for an $\varepsilon$ (and therefore in an equality). These relations capture the notion of a difference in only one entry of $\boldsymbol{\delta}$ or of $\varepsilon$, respectively. The choice of the inequality in the $\varepsilon \mathrm{s}$ and the $\delta \mathrm{s}$ is different. This is done to ensure that if either $(\boldsymbol{\delta}, \boldsymbol{\varepsilon}) \leq_{\mathcal{D}, i}\left(\boldsymbol{\delta}^{\prime}, \boldsymbol{\varepsilon}^{\prime}\right)$ or $(\boldsymbol{\delta}, \boldsymbol{\varepsilon}) \leq_{\mathcal{E}, i}\left(\boldsymbol{\delta}^{\prime}, \boldsymbol{\varepsilon}^{\prime}\right)$, then we have, for all formulas $\Phi$ over $f$, the inclusion

$$
\Gamma \mathrm{B}_{\delta, \varepsilon}(f, \Phi) \subseteq \Gamma \mathrm{B}_{\delta^{\prime}, \varepsilon^{\prime}}(f, \Phi)
$$

between the corresponding Gabrielov-Vorobjov Approximations. We write

$$
(\boldsymbol{\delta}, \boldsymbol{\varepsilon}) \stackrel{\mathcal{D}, i}{\rightsquigarrow}\left(\boldsymbol{\delta}^{\prime}, \boldsymbol{\varepsilon}^{\prime}\right)
$$

to denote that

$$
(\boldsymbol{\delta}, \boldsymbol{\varepsilon}) \leq_{\mathcal{D}, i}\left(\boldsymbol{\delta}^{\prime}, \boldsymbol{\varepsilon}^{\prime}\right) \quad \text { or } \quad\left(\boldsymbol{\delta}^{\prime}, \boldsymbol{\varepsilon}^{\prime}\right) \leq_{\mathcal{D}, i}(\boldsymbol{\delta}, \boldsymbol{\varepsilon})
$$

This notation is consistent with the meaning of updating $(\boldsymbol{\delta}, \boldsymbol{\varepsilon})$ to $\left(\boldsymbol{\delta}^{\prime}, \boldsymbol{\varepsilon}^{\prime}\right)$ by updating (either increasing or decreasing) only $\delta_{i}$ to $\delta_{i}^{\prime}$. We similarly define $(\boldsymbol{\delta}, \boldsymbol{\varepsilon}) \stackrel{\mathcal{E}, i}{\sim}\left(\boldsymbol{\delta}^{\prime}, \boldsymbol{\varepsilon}^{\prime}\right)$.

The following result states the main property of these rewritings.

Proposition 3.1. Let $f \in \mathcal{H}_{\boldsymbol{d}}[q], \Phi$ be a strict formula over $f$, and $\boldsymbol{\delta}, \boldsymbol{\delta}^{\prime}, \boldsymbol{\varepsilon}, \boldsymbol{\varepsilon}^{\prime} \in \mathbb{R}^{m}$ be such that both $(\boldsymbol{\delta}, \boldsymbol{\varepsilon})$ and $\left(\boldsymbol{\delta}^{\prime}, \boldsymbol{\varepsilon}^{\prime}\right)$ satisfy (2.4). If either $(\boldsymbol{\delta}, \boldsymbol{\varepsilon}) \underset{\sim}{\mathcal{D}, i}\left(\boldsymbol{\delta}^{\prime}, \boldsymbol{\varepsilon}^{\prime}\right)$ or $(\boldsymbol{\delta}, \boldsymbol{\varepsilon}) \stackrel{\mathcal{E}, i}{\sim}\left(\boldsymbol{\delta}^{\prime}, \boldsymbol{\varepsilon}^{\prime}\right)$, then the corresponding inclusion (3.1) of Gabrielov-Vorobjov Approximations induces a homotopy equivalence.

Proving Theorem 2.8 from this proposition is easy.

Proof of Theorem 2.8. By the definition of $\ll$, it is clear that there exist at least one $(\tilde{\boldsymbol{\delta}}, \tilde{\boldsymbol{\varepsilon}})$ satisfying both (2.3) and (2.4).

For any $(\boldsymbol{\delta}, \boldsymbol{\varepsilon})$ satisfying (2.4) , we can easily construct a sequence $\left(\boldsymbol{\delta}^{(0)}, \boldsymbol{\varepsilon}^{(0)}\right), \ldots,\left(\boldsymbol{\delta}^{(\ell)}, \boldsymbol{\varepsilon}^{(\ell)}\right)$ of pairs satisfying (2.4) such that

1. $\left(\boldsymbol{\delta}^{(0)}, \boldsymbol{\varepsilon}^{(0)}\right)=(\boldsymbol{\delta}, \boldsymbol{\varepsilon})$,

2. $\left(\boldsymbol{\delta}^{(\ell)}, \boldsymbol{\varepsilon}^{(\ell)}\right)=(\tilde{\boldsymbol{\delta}}, \tilde{\boldsymbol{\varepsilon}})$, and

3. for each $p \in\{0, \ldots, \ell-1\}$, there are $k_{p} \in\{\mathcal{D}, \mathcal{E}\}$ and $i_{p} \in\{1, \ldots, m\}$ such that

$$
\left(\boldsymbol{\delta}^{(p)}, \boldsymbol{\varepsilon}^{(p)}\right) \stackrel{k_{p}, i_{p}}{\sim}\left(\boldsymbol{\delta}^{(p+1)}, \boldsymbol{\varepsilon}^{(p+1)}\right) .
$$


For such a sequence, the isomorphism types of the homology groups of $\Gamma_{\boldsymbol{\delta}^{(p+1)}, \boldsymbol{\varepsilon}^{(p+1)}}(f, \Phi)$ don't change at each step as a consequence of Proposition 3.1. Thus $\Gamma_{\delta, \varepsilon}(f, \Phi)$ has homology groups isomorphic to those of $\Gamma_{\tilde{\tilde{\delta}}, \tilde{\boldsymbol{\varepsilon}}}(f, \Phi)$. The conclusion now follows from applying Theorem 2.4 to the latter.

We next focus on the situations $(\boldsymbol{\delta}, \boldsymbol{\varepsilon}) \stackrel{\mathcal{D}, i}{\sim}\left(\boldsymbol{\delta}^{\prime}, \boldsymbol{\varepsilon}^{\prime}\right)$ and $(\boldsymbol{\delta}, \boldsymbol{\varepsilon}) \stackrel{\mathcal{E}, i}{\underset{\sim}{\rightsquigarrow}}\left(\boldsymbol{\delta}^{\prime}, \boldsymbol{\varepsilon}^{\prime}\right)$. These situations correspond to replacing $\delta_{i}$ in the first one and $\varepsilon_{i}$ in the second one by some $\zeta \in\left(\varepsilon_{i}, \delta_{i}\right)$. Even though we are updating only one entry in the pair $(\boldsymbol{\delta}, \boldsymbol{\varepsilon})$, we have to modify the inequalities associated to several polynomials. Instead of doing this replacement simultaneously in all the inequalities, we do it by steps, in the inequalities corresponding to a single polynomial at a time. With this intuition at hand, we introduce the semialgebraic sets below.

Fix $f \in \mathcal{H}_{\boldsymbol{d}}[q]$, a strict formula $\Phi$ over $f$, positive numbers $\delta, \varepsilon, \zeta, t$, and $a \in\{0, \ldots, q\}$. We define the following spherical semialgebraic sets:

$\Gamma_{\delta, \varepsilon, \zeta, t}^{\mathcal{D}, a}(f, \Phi)$ is obtained from $\Phi$ by rewriting

$$
\begin{aligned}
& f_{l}=0 \rightsquigarrow\left|f_{l}(x)\right| \leq \varepsilon\left\|f_{l}\right\| \\
& f_{l}>0 \rightsquigarrow \begin{cases}f_{l}(x) \geq \delta\left\|f_{l}\right\| & \text { if } l>a \\
f_{a}(x) \geq t\left\|f_{a}\right\| & \text { if } l=a \\
f_{l}(x) \geq \zeta\left\|f_{l}\right\| & \text { if } l<a\end{cases} \\
& f_{l}<0 \rightsquigarrow \begin{cases}f_{l}(x) \leq-\delta\left\|f_{l}\right\| & \text { if } l>a \\
f_{a}(x) \leq-t\left\|f_{a}\right\| & \text { if } l=a \\
f_{l}(x) \leq-\zeta\left\|f_{l}\right\| & \text { if } l<a .\end{cases}
\end{aligned}
$$

$\Gamma_{\delta, \varepsilon, \zeta, t}^{\mathcal{E}, a}(f, \Phi)$ is obtained from $\Phi$ by rewriting

$$
\begin{aligned}
& f_{l}=0 \rightsquigarrow \begin{cases}\left|f_{l}(x)\right| \leq \varepsilon\left\|f_{l}\right\| & \text { if } l>a \\
\left|f_{a}(x)\right| \leq t\left\|f_{a}\right\| & \text { if } l=a \\
\left|f_{l}(x)\right| \leq \zeta\left\|f_{l}\right\| & \text { if } l<a\end{cases} \\
& f_{l}>0 \rightsquigarrow f_{l}(x) \geq \delta\left\|f_{l}\right\| \\
& f_{l}<0 \rightsquigarrow f_{l}(x) \leq-\delta\left\|f_{l}\right\| .
\end{aligned}
$$

Consider now $\boldsymbol{\delta}, \boldsymbol{\varepsilon} \in(0, \infty)^{m}, \mathfrak{c} \in\{\mathcal{D}, \mathcal{E}\}, i \in\{1, \ldots, m\}, a \in[q]$ and $\zeta, t>0$. We define the intermediate Gabrielov-Vorobjov approximations as the sets

$$
\Gamma \mathrm{B}_{\boldsymbol{\delta}, \varepsilon, \zeta, t}^{\mathfrak{c}, i, a}(f, \Phi):=\Gamma_{\delta_{i}, \varepsilon_{i}, \zeta, t}^{\mathfrak{c}, a}(f, \Phi) \cup \bigcup_{j \neq i} \Gamma_{\delta_{j}, \varepsilon_{j}}(f, \Phi) .
$$

In particular, we can see $\Gamma_{\boldsymbol{\delta}, \varepsilon, \zeta, t}^{\mathcal{D}, i, a}(f, \Phi)$ as the result of having replaced $\delta_{i}$ by $\zeta$ in all the inequalities with polynomials $f_{1}, \ldots, f_{a-1}$, and being in the process of making the replacement in those inequalities with $f_{a}$ with the parameter $t$ moving from $\delta_{i}$ to $\zeta$.

We now observe that for $\zeta, t, t^{\prime}>0$ with $t \leq t^{\prime}$ we have the inclusions

$$
\Gamma \mathrm{B}_{\boldsymbol{\delta}, \varepsilon, \zeta, t^{\prime}}^{\mathcal{D}, i, a}(f, \Phi) \subseteq \Gamma_{\boldsymbol{\delta}, \varepsilon, \zeta, t}^{\mathcal{D}, i, a}(f, \Phi) \quad \text { and } \quad \Gamma_{\boldsymbol{\delta}, \varepsilon, \zeta, t^{\prime}}^{\mathcal{E}, i, a}(f, \Phi) \supseteq \Gamma_{\boldsymbol{\delta}, \varepsilon, \zeta, t}^{\mathcal{E}, i, a}(f, \Phi)
$$

The crucial fact needed to prove Theorem 2.8 is that these inclusions induce homotopy equivalences. 
Proposition 3.2. Let $f \in \mathcal{H}_{\boldsymbol{d}}[q], \Phi$ be a strict formula, $\boldsymbol{\delta}, \boldsymbol{\varepsilon} \in \mathbb{R}^{m}$ satisfying (2.4), and let $i \in[m]$ and $a \in[q]$. Then,

(1) For all $\zeta \in\left(\varepsilon_{i}, \delta_{i}\right)$ and $\varepsilon_{i}<t \leq t^{\prime}<\varepsilon_{i+1}$ (where $\varepsilon_{m+1}=1 / \sqrt{2} \bar{\kappa}(f)$ by convention), the inclusion $\Gamma_{\boldsymbol{\delta}, \varepsilon, \zeta, t^{\prime}}^{\mathcal{D}, i, a}(f, \Phi) \subseteq \mathrm{B}_{\boldsymbol{\delta}, \varepsilon, \zeta, t}^{\mathcal{D}, i, a}(f, \Phi)$ induces a homotopy equivalence.

(2) For all $\zeta \in\left(\varepsilon_{i}, \delta_{i}\right)$ and $\delta_{i-1}<t \leq t^{\prime}<\delta_{i}$ (where $\delta_{0}=0$ by convention), the inclusion $\Gamma \mathrm{B}_{\boldsymbol{\delta}, \varepsilon, \zeta, t^{\prime}}^{\mathcal{E}, i, a}(f, \Phi) \supseteq \mathrm{B}_{\boldsymbol{\delta}, \varepsilon, \zeta, t}^{\mathcal{E}, i, a}(f, \Phi)$ induces a homotopy equivalence.

Again, Proposition 3.1 easily follows from this result.

Proof of Proposition 3.1. Assume that $(\boldsymbol{\delta}, \boldsymbol{\varepsilon}) \leq_{\mathcal{D}, i}\left(\boldsymbol{\delta}^{\prime}, \boldsymbol{\varepsilon}^{\prime}\right)$ holds. Then $\delta_{i} \geq \delta_{i}^{\prime}$ and without loss of generality, $\delta_{i}>\delta_{i}^{\prime}$. The following equalities then follow from the definition of $\Gamma_{\boldsymbol{\delta}, \varepsilon, \zeta, t}^{1, i, a}(f, \Phi)$ (we omit the $(f, \Phi)$ in what follows for simplicity):

- $\Gamma_{\boldsymbol{B}^{\prime}, \varepsilon, \delta_{i}^{\prime}, \delta_{i}}^{\mathcal{D}, i, 1}=\Gamma \mathrm{B}_{\delta, \varepsilon}$,

- $\Gamma \mathrm{B}_{\delta, \varepsilon, \delta_{i}^{\prime}, \delta_{i}^{\prime}}^{\mathcal{D}, i, a}=\Gamma \mathrm{B}_{\delta, \varepsilon, \delta_{i}^{\prime}, \delta_{i}}^{\mathcal{D}, i, a+1}, \quad$ for all $a \in[q-1]$,

- $\Gamma \mathrm{B}_{\boldsymbol{\delta}, \varepsilon, \delta_{i}^{\prime}, \delta_{i}^{\prime}}^{\mathcal{D}, i,}=\Gamma \mathrm{B}_{\boldsymbol{\delta}^{\prime}, \varepsilon}=\Gamma \mathrm{B}_{\boldsymbol{\delta}^{\prime}, \varepsilon^{\prime}}$,

the last equality as, by assumption, $\varepsilon=\varepsilon^{\prime}$. These equalities yield the following chain

$$
\Gamma \mathrm{B}_{\boldsymbol{\delta}, \varepsilon}=\Gamma \mathrm{B}_{\boldsymbol{\delta}, \boldsymbol{\varepsilon}, \delta_{i}^{\prime}, \delta_{i}}^{\mathcal{D}, i, 1} \subseteq \Gamma \mathrm{B}_{\boldsymbol{\delta}, \varepsilon, \delta_{i}^{\prime}, \delta_{i}^{\prime}}^{\mathcal{D}, i, 1}=\Gamma \mathrm{B}_{\boldsymbol{\delta}, \varepsilon, \delta_{i}^{\prime}, \delta_{i}}^{\mathcal{D}, i, 2} \subseteq \Gamma \mathrm{B}_{\boldsymbol{\delta}, \varepsilon, \delta_{i}^{\prime}, \delta_{i}^{\prime}}^{\mathcal{D}, i, 2}=\cdots \subseteq \Gamma \mathrm{B}_{\boldsymbol{\delta}, \varepsilon, \delta_{i}^{\prime}, \delta_{i}^{\prime}}^{\mathcal{D}, i, q}=\Gamma_{\boldsymbol{\delta}^{\prime}, \boldsymbol{\varepsilon}^{\prime}}
$$

in which all inclusions induce homotopy equivalences by Proposition 3.2 (1). Hence Proposition 3.1 follows in this case.

For the other cases, i.e., when $\left(\boldsymbol{\delta}^{\prime}, \boldsymbol{\varepsilon}^{\prime}\right) \leq_{\mathcal{D}, i}(\boldsymbol{\delta}, \boldsymbol{\varepsilon})$ or when $(\boldsymbol{\delta}, \boldsymbol{\varepsilon}) \stackrel{\mathcal{E}, i}{\rightsquigarrow}\left(\boldsymbol{\delta}^{\prime}, \boldsymbol{\varepsilon}^{\prime}\right)$, we proceed analogously.

\subsection{Some elements of differential topology}

To prove Proposition 3.2 will require more elaborate arguments. We lay down these arguments in this section. To do so, we first review the basic notions of the Mather-Thom theory, already introduced in [2], now using some more general results from [9]. We then develop a generalization of the sign partition, called $(f, \boldsymbol{\lambda})$-partition. We will show that these new partitions, under well-posedness assumptions on $f$, are Whitney stratifications.

\subsubsection{Stratified sets and Mather-Thom theory}

We will take a more general approach than that in [2], as this time we will consider Whitney stratifications for more general sets. For motivation, however, we refer to [2, §4.1].

Definition 3.3. [11, 9] A Whitney stratification of a subset $\Omega$ of a smooth manifold $\mathcal{M}$ of dimension $m$ is a partition $\mathcal{S}$ of $\Omega$ into locally closed smooth submanifolds of $\mathcal{M}$, called strata, such that:

F (Locally finite) Every $x \in \Omega$ has a neighborhood intersecting finitely many strata only. 
W (Whitney's condition b) For every strata $\varsigma, \sigma \in \mathcal{S}$, every point $x \in \varsigma \cap \bar{\sigma}$, every sequence of points $\left\{x_{\ell}\right\}_{\ell \in \mathbb{N}}$ in $\varsigma$ converging to $x$, and every sequence of points $\left\{y_{\ell}\right\}_{\ell \in \mathbb{N}}$ in $\sigma$ converging to $x$, we have that, in all local charts of $\mathcal{M}$ around $x$,

$$
\lim _{\ell \rightarrow \infty} \overline{x_{\ell}, y_{\ell}} \subseteq \lim _{\ell \rightarrow \infty} \mathrm{T}_{y_{\ell}} \sigma,
$$

provided both limits exist. The inclusion should be interpreted in the local coordinates of the chart: $\overline{x_{\ell}, y_{\ell}}$ denotes the straight line joining $x_{\ell}$ and $y_{\ell}, \mathrm{T}_{y_{\ell}} \sigma$ denotes the affine plane tangent to $\sigma$ at $y_{\ell}$, and the limits are to be interpreted in the corresponding Grassmannians of $\mathbb{R}^{m}$.

Recall that a map is proper when its inverse image of any compact subset is compact. Our interest on Whitney stratified sets is linked to the following result, a version of the so-called Thom's First Isotopy Lemma (see [9, Ch. II, (5.2)]).

Theorem 3.4. Let $\mathcal{M}$ be a smooth manifold with a Whitney stratification $\mathcal{S}$ of a locally closed subset $\Omega \subseteq \mathcal{M}$ and let $\alpha: \mathcal{M} \rightarrow \mathbb{R}^{k}$ be a smooth map such that:

(i) $\alpha: \Omega \rightarrow \mathbb{R}^{k}$ is proper,

(ii) $\alpha_{\mid \sigma}: \sigma \rightarrow \mathbb{R}^{k}$ is a surjective submersion, for each stratum $\sigma \in \mathcal{S}$.

Then $\alpha: \Omega \rightarrow \mathbb{R}^{k}$ is a trivial fiber bundle. That is, there exist a subset $F \subseteq \Omega$ and a homeomorphism $h=\left(h_{\mathbb{R}^{k}}, h_{F}\right): \Omega \rightarrow \mathbb{R}^{k} \times F$ such that $\alpha=h_{\mathbb{R}^{k}}$. Furthermore, for all $x, y \in \Omega, h_{F}(x)=h_{F}(y)$ implies that $x$ and $y$ lie in the same stratum of $\mathcal{S}$.

Remark 3.5. As the codomain of $\alpha$ is $\mathbb{R}^{k}$, it follows from the proof of [9, Ch. II, (5.2)] that we have a trivial fiber bundle and not just a locally trivial fiber bundle. The last sentence follows from noting that the trivial fibration in the statement of [9, Ch. II, (5.2)] is stratified, see [9, Ch. II, (5.1)].

\subsection{2 $(f, \lambda)$-partitions}

A $q$-tuple $f \in \mathcal{H}_{\boldsymbol{d}}[q]$ of polynomials leads to partition of the sphere $\mathbb{S}^{n}$ according to the $q$-tuples of signs obtained when evaluating the polynomials. We are going to make a finer classification by considering not only the signs, but also the magnitudes of the values with respect to some finite grid. This is the idea of $(f, \boldsymbol{\lambda})$-partitions.

Definition 3.6. Let $f \in \mathcal{H}_{\boldsymbol{d}}[q]$ and $\boldsymbol{\lambda} \in \mathbb{R}^{q \times(m+1)}$ be a matrix whose entries satisfy $0=\lambda_{i, 0}<\lambda_{i, 1}<\cdots<\lambda_{i, m}$, for each $i \in[q]$. To each point $x \in \mathbb{S}^{n}$ we associate the following sets:

$(\mathrm{I} \bullet)$ For all $0 \leq k \leq m, I_{\bullet, k}(x):=\left\{i \in[q]|| f_{i}(x) \mid /\left\|f_{i}\right\|=\lambda_{i, k}\right\}$.

(I\1) For all $0 \leq k<m, I_{\chi, k}(x):=\left\{i \in[q]\left|\lambda_{i, k}<\right| f_{i}(x) \mid /\left\|f_{i}\right\|<\lambda_{i, k+1}\right\}$.

(I\2) $I_{\chi, m}(x):=\left\{i \in[q]\left|\lambda_{i, m}<\right| f_{i}(x) \mid /\left\|f_{i}\right\|\right\}$.

This defines the ordered partition of $[q]$ (in which we allow empty sets):

$$
\boldsymbol{I}(x):=\left(I_{\bullet, 0}(x), I_{\chi, 0}(x), I_{\bullet, 1}(x), I_{\chi, 1}(x), \ldots, I_{\bullet, m-1}(x), I_{\chi, m-1}(x), I_{\bullet}, m(x), I_{\chi, m}(x)\right) .
$$

The point $x$ also determines the tuple of sign conditions $\sigma(x) \in\{-1,0,+1\}^{q}$ given by 
(S) $\sigma_{i}(x):=\operatorname{sgn}\left(f_{i}(x)\right)$ for $i \in[q]$.

It is clear that

$$
x \sim y:=(\boldsymbol{I}(x)=\boldsymbol{I}(y) \text { and } \sigma(x)=\sigma(y))
$$

describes an equivalence relation. We define the $(f, \boldsymbol{\lambda})$-partition $\Pi_{f, \boldsymbol{\lambda}}$ as the set of equivalence classes of this relation.

An ordered partition $\boldsymbol{I}:=\left(I_{\bullet, 0}, I_{\chi, 0}, \ldots, I_{\bullet, m}, I_{\ell, m}\right)$ of $[q]$ together with a sign vector $\sigma \in\{-1,0,+1\}^{q}$ defines the set

$$
\Pi_{\boldsymbol{I}, \sigma}:=\left\{x \in \mathbb{S}^{n} \mid \boldsymbol{I}(x)=\boldsymbol{I}, \sigma(x)=\sigma\right\},
$$

which is an element of $\Pi_{f, \boldsymbol{\lambda}}$, provided it is non-empty.

Remark 3.7. Less formally, the construction of $\Pi_{f, \boldsymbol{\lambda}}$ can be described as follows: the $i$ th row of the matrix $\boldsymbol{\lambda} \in \mathbb{R}^{q \times(m+1)}$ defines a partition of the set $\mathbb{R}_{\geq 0}$ of nonnegative reals into the open intervals $\left(\lambda_{i, k}, \lambda_{i, k+1}\right)$ and the singleton sets $\left\{\lambda_{i, k}\right\}$. By considering also the numbers $\pm \lambda_{i, k}$, we similarly obtain a partition of $\mathbb{R}$, which is symmetric with respect to the reflection $x \mapsto-x$. The product of these partitions of $\mathbb{R}$, for $i \in[q]$, yields the partition $\Pi_{f, \boldsymbol{\lambda}}$ of $\mathbb{R}^{q}$. So the sets of this partition are products of open intervals and singletons, describing where a value $\left(y_{1}, \ldots, y_{q}\right) \in \mathbb{R}^{q}$ is located within the discrete grid provided by the matrix $\boldsymbol{\lambda}$

Example 3.8. Figure 4 shows, locally, an example on $\mathbb{S}^{2}$ with $q=2, m=2$ and $\lambda_{1, i}=$ $\lambda_{2, i}=\lambda_{i}$. The thick curves correspond to the zero sets for $f_{1}$ and $f_{2}$. The dashed lines are level curves (for both $f_{1}$ and $f_{2}$ ) with levels $-\lambda_{1}$ and $\lambda_{1}$ and the dotted curves are the same for the levels $-\lambda_{2}$ and $\lambda_{2}$. All these curves partition the picture into 36 two-dimensional open regions, 60 open segments, and 25 points. Each of these 121 regions corresponds to an element in $\Pi_{f, \boldsymbol{\lambda}}$. We won't attempt to write down the details for each of them but in Table 1 we list some of them with their corresponding ordered partition.

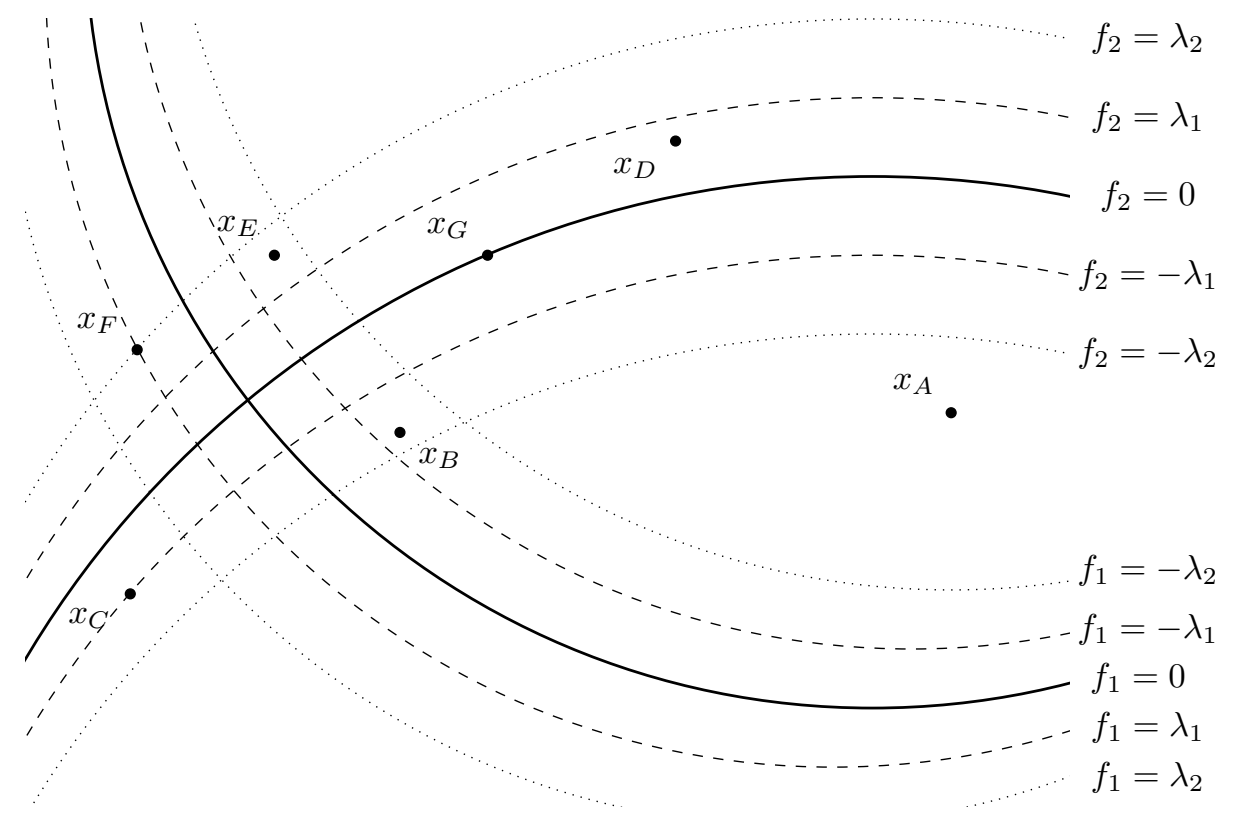

Figure 4 An example (locally) on $\mathbb{S}^{2}$ with $S=\{1,2\}$ and $m=2$. 


\begin{tabular}{|c|c|c|c|c|c|c|c|}
\hline \hline & $I_{\bullet, 0}$ & $I_{\chi_{, 0}}$ & $I_{\bullet, 1}$ & $I_{\varnothing, 1}$ & $I_{\bullet, 2}$ & $I_{\varnothing, 2}$ & $\sigma$ \\
\hline$x_{A}$ & $\varnothing$ & $\varnothing$ & $\varnothing$ & $\varnothing$ & $\varnothing$ & $\{1,2\}$ & $(-1,-1)$ \\
$x_{B}$ & $\varnothing$ & $\varnothing$ & $\varnothing$ & $\{1,2\}$ & $\varnothing$ & $\varnothing$ & $(-1,-1)$ \\
$x_{C}$ & $\varnothing$ & $\varnothing$ & $\varnothing$ & $\varnothing$ & $\{2\}$ & $\{1\}$ & $(+1,-1)$ \\
$x_{D}$ & $\varnothing$ & $\{2\}$ & $\varnothing$ & $\varnothing$ & $\varnothing$ & $\{1\}$ & $(-1,+1)$ \\
$x_{E}$ & $\varnothing$ & $\varnothing$ & $\varnothing$ & $\{1,2\}$ & $\varnothing$ & $\varnothing$ & $(-1,+1)$ \\
$x_{F}$ & $\varnothing$ & $\varnothing$ & $\{1\}$ & $\varnothing$ & $\{2\}$ & $\varnothing$ & $(+1,+1)$ \\
$x_{G}$ & $\{2\}$ & $\varnothing$ & $\varnothing$ & $\varnothing$ & $\varnothing$ & $\{1\}$ & $(-1,0)$ \\
\hline \hline
\end{tabular}

Table 1 Some points in Figure 4 and their ordered partition.

The following theorem gives sufficient conditions on $f \in \mathcal{H}_{\boldsymbol{d}}[q]$ and $\boldsymbol{\lambda} \in \mathbb{R}^{q \times(m+1)}$ for the $(f, \boldsymbol{\lambda})$-partition of $\mathbb{S}^{n}$ to be a Whitney stratification.

Theorem 3.9. Let $f \in \mathcal{H}_{\boldsymbol{d}}[q]$ with $\bar{\kappa}(f)<\infty$ and assume $\boldsymbol{\lambda} \in \mathbb{R}^{q \times(m+1)}$ satisfies for $i \in[q]$,

$$
0=\lambda_{i, 0}<\lambda_{i, 1}<\cdots<\lambda_{i, m}<\frac{1}{\sqrt{2} \bar{\kappa}(f)}
$$

Then the $(f, \boldsymbol{\lambda})$-partition $\Pi_{f, \boldsymbol{\lambda}}$ is a Whitney stratification of $\mathbb{S}^{n}$. Furthermore, under these conditions, the following holds:

(1) The codimension in $\mathbb{S}^{n}$ of each stratum $\Pi_{\boldsymbol{I}, \sigma}$ equals $\sum_{k=0}^{m}\left|I_{\bullet, k}\right|=q-\sum_{k=0}^{m}\left|I_{\searrow, k}\right|$.

(2) Given $\Pi_{\boldsymbol{I}, \sigma} \in \Pi_{f, \boldsymbol{\lambda}}$ and $a \in I_{\chi, k}$ for some $k<m$, the map

$$
\begin{aligned}
\hat{f}_{a, \boldsymbol{I}, \sigma}: \Pi_{\boldsymbol{I}, \sigma} & \rightarrow\left(\lambda_{a, k}, \lambda_{a, k+1}\right) \\
x & \mapsto\left|f_{a}(x)\right| /\left\|f_{a}\right\|
\end{aligned}
$$

is a surjective submersion.

Remark 3.10. Recall that the condition " $a \in I_{\chi, k}$ for some $k<m$ " can be less cryptically written as " $\left|f_{a}(x)\right| /\left\|f_{a}\right\| \in\left(\lambda_{a, k}, \lambda_{a, k+1}\right)$ for some $x \in \Pi_{\boldsymbol{I}, \sigma}$ and $k<m$ ", or simply as " $\left|f_{a}\right| /\left\|f_{a}\right\| \in\left(\lambda_{a, k}, \lambda_{a, k+1}\right)$ on $\Pi_{\boldsymbol{I}, \sigma}$ for some $k<m$ ".

Proof. In order to show that $\Pi_{f, \boldsymbol{\lambda}}$ is a Whitney stratification, we notice that

$$
\Pi_{f, \boldsymbol{\lambda}}=\bigwedge_{i=1}^{q} \Pi_{f_{i}, \lambda_{i}}:=\left\{\cap_{i=1}^{q} \Pi_{i} \mid \Pi_{i} \in \Pi_{f_{i}, \lambda_{i}}\right\}
$$

where $\lambda_{i}:=\left(\lambda_{i, 0}, \ldots, \lambda_{i, m}\right)$ is the $i$ th row of $\boldsymbol{\lambda}$ and $\Pi_{f_{i}, \lambda_{i}}$ is the $\left(f_{i}, \lambda_{i}\right)$-partition of $\mathbb{S}^{n}$. Thus, in virtue of $\left[9\right.$, Ch. I, (1.3)], it is enough to show that each $\Pi_{f_{i}, \lambda_{i}}$ is a Whitney stratification and that $\Pi_{f_{1}, \lambda_{1}}, \ldots, \Pi_{f_{q}, \lambda_{q}}$ are in general position, which, following [9, Ch. I, (1.2)], means that for all $\Pi_{1} \in \Pi_{f_{1}, \lambda_{1}}, \ldots, \Pi_{q} \in \Pi_{f_{q}, \lambda_{q}}$,

$$
\operatorname{codim} \bigcap_{i=1}^{q} \mathrm{~T}_{x} \Pi_{i}=\sum_{i=1}^{q} \operatorname{codim} \mathrm{T}_{x} \Pi_{i},
$$

holds for each $x \in \bigcap_{i=1}^{q} \Pi_{i}$, where codim stands for codimension. 
Note that $\Pi_{f_{i}, \lambda_{i}}$ consists of open sets of the form $f_{i}^{-1}(a, b)$, with $(a, b)$ an open interval, or a hypersurface of the form $f_{i}^{-1}(t)$, with $t=\left\|f_{i}\right\| \lambda_{i, j}$ for some $j$. By assumption on $\boldsymbol{\lambda}$, this implies that for such $t,|t|<\left\|f_{i}\right\| /(\sqrt{2} \bar{\kappa}(f))$ and hence, by [2, Proposition 3.6] and the implicit function theorem, all the hypersurfaces are smooth. Whitney's condition b is verified in a straighforward way so that we conclude that $\Pi_{f_{i}, \lambda_{i}}$ is a Whitney stratification.

We show now that $\Pi_{f_{1}, \lambda_{1}}, \ldots, \Pi_{f_{q}, \lambda_{q}}$ are in general position. Let $\Pi_{i} \in \Pi_{f_{i}, \lambda_{i}}$, for $i \in[q]$, and $x \in \cap_{i \leq q} \Pi_{i}$. It is easy to check that codim $\mathrm{T}_{x} \Pi_{i}=1$ if $i \in I_{\bullet}, k(x)$ and $\operatorname{codim} \mathrm{T}_{x} \Pi_{i}=$ 0 otherwise. Therefore, abbreviating $I_{\bullet}, *(x):=\bigcup_{k} I_{\bullet, k}(x)$, we get $\sum_{i=1}^{q} \operatorname{codim} \mathrm{T}_{x} \Pi_{i}=$ $\left|I_{\bullet}, *(x)\right|$. In addition, when $\Pi_{i}$ is a hypersurface, we have $\mathrm{T}_{x} \Pi_{i}=\operatorname{ker} \mathrm{D}_{x} f_{i}$, thus

$$
\bigcap_{i=1}^{q} \mathrm{~T}_{x} \Pi_{i}=\bigcap_{i \in I_{*}, 0} \operatorname{ker} \mathrm{D}_{x} f_{i}=\operatorname{ker} \mathrm{D}_{x} f^{I_{\bullet}, *}(x) .
$$

By [2, Proposition 3.6], the codimension of the right-hand side is $\left|I_{\bullet}, *(x)\right|$. This shows that $\Pi_{f_{1}, \lambda_{1}}, \ldots, \Pi_{f_{q}, \lambda_{q}}$ are in general position. We conclude that $\Pi_{f, \boldsymbol{\lambda}}$ is a Whitney stratification.

The argument above proves also (1).

We prove part (2) is a standard way. First, we will show that $\hat{f}_{a, \boldsymbol{I}, \sigma}$ is a submersion, i.e., that its gradient is not tangent to $\Pi_{\boldsymbol{I}, \sigma}$. Then we will show that $\hat{f}_{a, \boldsymbol{I}, \sigma}$ is closed. Assume we have proved these claims. The fact that $\hat{f}_{a, \boldsymbol{I}, \sigma}$ is a submersion implies that it is open. Therefore, as $\hat{f}_{a, \boldsymbol{I}, \sigma}$ is also closed and $\left(\lambda_{i, k}, \lambda_{i, k+1}\right)$ is connected, we deduce that $\hat{f}_{a, \boldsymbol{I}, \sigma}$ is surjective. This will finish the proof.

To show that $\hat{f}_{a, \boldsymbol{I}, \sigma}$ is a submersion, we fix a point $p \in \Pi_{\boldsymbol{I}, \sigma}$ and take trivializing coordinates around it, using [2, Lemma 4.24]. In these coordinates, using the notation from [2], $\Pi_{\boldsymbol{I}, \sigma}$ is an open subset of an affine subspace given by

$$
\begin{cases}U_{i}=\sigma_{i} \lambda_{i, k} & \left(0<k \leq m, i \in I_{\bullet, k}\right) \\ \lambda_{i, k} \leq \sigma_{i} U_{i} \leq \lambda_{i, k+1} & \left(0 \leq k<m, l \geq 1, i \in I_{\chi, k}\right)\end{cases}
$$

whose tangent space is given by the system

$$
\left\{U_{i}=0 \quad\left(0 \leq k \leq m, i \in I_{\bullet}, k\right) .\right.
$$

The map $\hat{f}_{a, \boldsymbol{I}, \sigma}$ in these coordinates becomes the linear map $U_{a}$. To check that $\hat{f}_{a, \boldsymbol{I}, \sigma}$ is a submersion is then enough to check that $U_{a}$ is not identically zero in the tangent space in these coordinates. Since $a \notin \cup_{k} I_{\bullet}, k, U_{a}$, this is the case and so $\hat{f}_{a, \boldsymbol{I}, \sigma}$ is a submersion.

To show that $\hat{f}_{a, \boldsymbol{I}, \sigma}$ is closed, it is enough to show that for every sequence $\left\{x_{k}\right\}$ in $\Pi_{\boldsymbol{I}, \sigma}$, if $\left\{\hat{f}_{a, \boldsymbol{I}, \sigma}\left(x_{k}\right)\right\}$ has a limit $\lambda \in\left(\lambda_{a, k}, \lambda_{a, k+1}\right)$, then there exists $x \in \Pi_{\boldsymbol{I}, \sigma}$ such that $\hat{f}_{a, \boldsymbol{I}, \sigma}(x)=\lambda$.

As $\mathbb{S}^{n}$ is compact, we can assume without loss of generality that $\left\{x_{k}\right\}$ converges to a point $x^{\prime} \in \overline{\Pi_{\boldsymbol{I}, \sigma}}$. By continuity, $\hat{f}_{a, \boldsymbol{I}, \sigma}\left(x^{\prime}\right)=\lambda$. Passing again to trivializing coordinates and using [2, Lemma 4.24], we perturb $x^{\prime}$ to a point $x$ whose components in these coordinates are as follows:

$$
u_{i}:= \begin{cases}u_{i}^{\prime}+\sigma_{i} t & \text { if for some } k \text { we have } i \in I_{k, 1} \text { and } u_{i}^{\prime}=\lambda_{i, k} \\ u_{i}^{\prime}-\sigma_{i} t & \text { if for some } k \text { we have } i \in I_{k, 1} \text { and } u_{i}^{\prime}=\lambda_{i, k+1} \\ u_{i}^{\prime} & \text { otherwise }\end{cases}
$$


with a sufficiently small $t>0$. This new point $x$ evaluates to the same value as $x^{\prime}$ under $\hat{f}_{a, \boldsymbol{I}, \sigma}$, since $u_{a}=u_{a}^{\prime}$ as $u_{a}^{\prime}=\lambda \in\left(\lambda_{a, k}, \lambda_{a, k+1}\right)$ by hypothesis; and it belongs to $\Pi_{\boldsymbol{I}, \sigma}$. Thus it is the desired point and we are done.

\subsection{Proof of Proposition 3.2}

We have now all the tools needed to prove Proposition 3.2 and with it to finish the proof of the Quantitative Gabrielov-Vorobjov Theorem 2.8, We will only prove part (1) of Proposition 3.2 as part (2) is proven in an analogous way.

We fix $f \in \mathcal{H}_{\boldsymbol{d}}[q]$, a strict formula $\Phi$ over $f$, tuples $\boldsymbol{\delta}, \boldsymbol{\varepsilon} \in(0, \infty)^{m}$, an index $i \in[m]$, a point $\zeta \in\left(\varepsilon_{i}, \delta_{i}\right)$, points $t<t^{\prime}$ in the interval $\left(\varepsilon_{i}, \varepsilon_{i+1}\right)$, and an index $a \in[q]$, as in the statement of Proposition 3.2 and satisfying the hypothesis given there. Since $a$ is fixed, we can assume $\left\|f_{a}\right\|=1$ without loss of generality after scaling $f$ appropriately.

We also choose positive numbers $t_{0}, t_{1}$ satisfying

$$
\varepsilon_{i}<t_{0}<t<t^{\prime}<t_{1}<\varepsilon_{i+1}
$$

and define the matrix $\boldsymbol{\lambda} \in \mathbb{R}^{q \times(2 m+2)}$ whose $l$ th row $\lambda_{l}$ is given by

$$
\lambda_{l}:= \begin{cases}\left(0, \varepsilon_{1}, \delta_{1}, \ldots, \varepsilon_{i}, \zeta, \delta_{i}, \varepsilon_{i+1} \ldots, \varepsilon_{m}, \delta_{m}\right), & \text { if } l \neq a, \\ \left(0, \varepsilon_{1}, \delta_{1}, \ldots, \varepsilon_{i}, t_{0}, t_{1}, \varepsilon_{i+1}, \ldots, \varepsilon_{m}, \delta_{m}\right), & \text { if } l=a .\end{cases}
$$

By construction, this $\boldsymbol{\lambda}$ satisfies (3.4). We will assume these conventions throughout this subsection without further mentioning them explicitly. The matrix $\boldsymbol{\lambda}$ determines the $(f, \boldsymbol{\lambda})$ partition $\Pi_{f, \boldsymbol{\lambda}}$ which, as we saw in Theorem 3.9, is a Whitney stratification of $\mathbb{S}^{n}$.

Recall the intermediate Gabrielov-Vorobjov approximations

$$
\Gamma \mathrm{B}_{\tau}:=\Gamma \mathrm{B}_{\boldsymbol{\delta}, \varepsilon, \zeta, \tau}^{\mathcal{D}, i, a}(f, \Phi),
$$

defined in (3.2), for $\tau \in\left[t_{0}, t_{1}\right)$. These are compact subsets of $\mathbb{S}^{n}$.

Proposition 3.2 claims that $\iota: \Gamma \mathrm{B}_{t^{\prime}} \rightarrow \Gamma \mathrm{B}_{t}$ is a homotopy equivalence. The basic idea for showing this is to apply Theorem 3.4 to the stratification provided by $\Pi_{f, \lambda}$. In a first step towards this goal, we describe how the strata $\Pi_{\boldsymbol{I}, \sigma}$ of $\Pi_{f, \boldsymbol{\lambda}}$ intersect $\Gamma_{\tau}$. The findings are summarized in the proposition below, whose easy but somewhat cumbersome proof is postponed to 33.3 .2 .

Proposition 3.11. (1) $\Gamma_{t_{0}}$ is a union of strata of $\Pi_{f, \lambda}$.

(2) Let $\tau, \tau^{\prime} \in\left(t_{0}, t_{1}\right)$. For each $\Pi_{\boldsymbol{I}, \sigma} \in \Pi_{f, \boldsymbol{\lambda}}$ such that $\Pi_{\boldsymbol{I}, \sigma} \subseteq \Gamma_{t_{0}}$, the following holds:

(i) $\Pi_{\boldsymbol{I}, \sigma} \cap \Gamma_{\tau}=\varnothing$ if and only if $\Pi_{\boldsymbol{I}, \sigma} \cap \Gamma_{\tau^{\prime}}=\varnothing$. In this case, $\Pi_{\boldsymbol{I}, \sigma} \subseteq\left|f_{a}\right|^{-1}\left(t_{0}\right)$.

(ii) $\Pi_{\boldsymbol{I}, \sigma} \cap \Gamma_{\tau}=\Pi_{\boldsymbol{I}, \sigma}$ if and only if $\Pi_{\boldsymbol{I}, \sigma} \cap \Gamma_{\tau^{\prime}}=\Pi_{\boldsymbol{I}, \sigma}$.

(iii) If $\varnothing \neq \Pi_{\boldsymbol{I}, \sigma} \cap \Gamma_{\tau} \varsubsetneqq \Pi_{\boldsymbol{I}, \sigma}$, then $\Pi_{\boldsymbol{I}, \sigma} \subseteq\left|f_{a}\right|^{-1}\left(t_{0}, t_{1}\right)$ and

$$
\Pi_{\boldsymbol{I}, \sigma} \cap \Gamma_{\tau}=\Pi_{\boldsymbol{I}, \sigma} \cap\left\{x \in \mathbb{S}^{n}|| f_{a}(x) \mid \geq \tau\right\} .
$$




\subsubsection{Homotopies preserving $\Pi_{f, \lambda}$}

We are now going to construct the maps and homotopies to show that the inclusion $\iota: \Gamma \mathrm{B}_{t^{\prime}} \rightarrow \Gamma \mathrm{B}_{t}$ is a homotopy equivalence. For this, we should construct a continuous map $\rho: \Gamma \mathrm{B}_{t} \rightarrow \Gamma \mathrm{B}_{t^{\prime}}$ and homotopies between the compositions of these maps and the identity maps.

A first approach would be to move around the points of $\Gamma \mathrm{B}_{t} \backslash \Gamma \mathrm{B}_{t^{\prime}}$ and then extend the maps obtained continuously to the whole space. It is easier though to work in the larger space $\Gamma \mathrm{B}_{t_{0}} \cap\left|f_{a}\right|^{-1}\left(t_{0}, t_{1}\right)$, where we can control what happens at the boundary and thus obtain the continuous extensions.

Consider the open subset $\mathcal{M}:=\mathbb{S}^{n} \backslash f_{a}^{-1}(0)$ of $\mathbb{S}^{n}$ together with the smooth map $\mathcal{M} \rightarrow \mathbb{R}, s \mapsto\left|f_{a}(x)\right|$, as well as the locally closed set

$$
\Omega:=\Gamma_{t_{0}} \cap\left|f_{a}\right|^{-1}\left(t_{0}, t_{1}\right) \subseteq \mathcal{M} .
$$

By Proposition $3.11(1), \Omega$ is the union of certain strata $\Pi_{\boldsymbol{I}, \sigma}$ of $\Pi_{f, \boldsymbol{\lambda}}$, namely of those strata on which $\left|f_{a}\right|$ takes values in $\left(t_{0}, t_{1}\right)$. We note that the restriction of $\left|f_{a}\right|$,

$$
\alpha: \Omega \rightarrow\left(t_{0}, t_{1}\right), x \mapsto\left|f_{a}(x)\right|,
$$

is a proper map. Indeed, the inverse image $\alpha^{-1}(J)=\left\{x \in \Gamma \mathrm{B}_{t_{0}} \mid f_{a}(x) \in J\right\}$ of a compact subset $J \subseteq\left(t_{0}, t_{1}\right)$ is a closed subset of the compact set $\Gamma_{t_{0}}$ and thus compact itself.

By Theorem 3.9, $\Pi_{f, \boldsymbol{\lambda}}$ restricts to a Whitney stratification of $\Omega$ and the map $\alpha$ satisfies the hypothesis of Theorem 3.4. Therefore, there is a subset $F \subseteq \Omega$ and a homeomorphism $h: \Omega \rightarrow F \times\left(t_{0}, t_{1}\right)$ such that the following diagram commutes

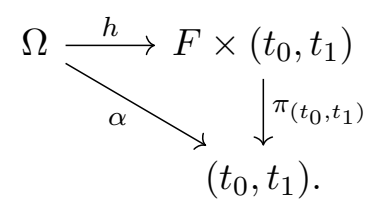

Moreover, the stratum in which $x \in \Omega$ lies only depends on $h_{F}(x)$, that is, if $h_{F}(x)=h_{F}(y)$ then $x$ and $y$ belong to the same stratum of $\Pi_{f, \lambda}$.

Consider the following continuous (piecewise linear) map

$$
\begin{aligned}
v:[0,1] \times\left[t_{0}, t_{1}\right] & \rightarrow\left[t_{0}, t_{1}\right] \\
(s, y) & \mapsto \begin{cases}y & \text { if } y \in\left[t^{\prime}, t_{1}\right], \\
(1-s) y+s t^{\prime} & \text { if } y \in\left[t, t^{\prime}\right], \\
\frac{t_{0}+t}{2}+\left((1-s)+s \frac{2 t^{\prime}-t-t_{0}}{t-t_{0}}\right)\left(y-\frac{t_{0}+t}{2}\right) & \text { if } y \in\left[\left(t_{0}+t\right) / 2, t\right], \\
y & \text { if } y \in\left[t_{0},\left(t_{0}+t\right) / 2\right] .\end{cases}
\end{aligned}
$$

One easily verifies that this map restricts to a continuous retraction of $\left[t, t_{1}\right]$ onto $\left[t^{\prime}, t_{1}\right]$ that leaves fixed all points in a neighborhood of $\left\{t_{0}, t_{1}\right\}$. With the help of $v$, one defines the continuous map

$$
\begin{aligned}
\psi:[0,1] \times \Omega & \rightarrow \Omega \\
(s, x) & \mapsto \begin{cases}x, & \text { if } \alpha(x) \notin\left(\left(t_{0}+t\right) / 2,\left(t_{1}+t^{\prime}\right) / 2\right), \\
h^{-1}\left(h_{F}(x), v(s, \alpha(x))\right), & \text { otherwise. }\end{cases}
\end{aligned}
$$


The properties of $v$ and $h$ imply that this map restricts to a continuous retraction of $\alpha^{-1}\left[t, t_{1}\right)$ onto $\alpha^{-1}\left[t^{\prime}, t_{1}\right)$ that leaves fixed all points in a neighborhood of the boundary $\partial \Omega \cap \Gamma \mathrm{B}_{t_{0}}$ of $\Omega$ in $\Gamma_{t_{0}}\left(\right.$ note $\partial \Omega \subseteq\left|f_{a}\right|^{-1}\left(\left\{t_{0}, t_{1}\right\}\right)$ ).

We also have that $\psi\left(s, \Pi_{\boldsymbol{I}, \sigma}\right) \subseteq \Pi_{\boldsymbol{I}, \sigma}$ for all $s \in[0,1]$, provided $\Pi_{\boldsymbol{I}, \sigma} \subseteq \Omega$. This is so, because the value $h_{F}(x)$ determines the stratum to which $x$ belongs and moreover $h_{F}(\psi(s, x))=h_{F}(x)$.

Since $\psi$ fixes all points in a neighborhood of $\partial \Omega \cap \Gamma \mathrm{B}_{t_{0}}$, it can be extended to the continuous map $\Psi:[0,1] \times \Gamma \mathrm{B}_{t_{0}} \rightarrow \Gamma \mathrm{B}_{t_{0}}$ given by

$$
\Psi(s, x)= \begin{cases}\psi(s, x), & \text { if } x \in \Omega, \\ x, & \text { otherwise }\end{cases}
$$

As we are extending by the identity, all properties of $\psi$ are inherited by $\Psi$. In other words, $\Psi$ restricts to a continuous retraction of $\Gamma_{t_{0}} \cap\left|f_{a}\right|^{-1}[t, \infty)=\left(\Gamma_{t_{0}} \backslash \Omega\right) \cup \alpha^{-1}\left[t, t_{1}\right)$ onto $\Gamma_{t_{0}} \cap\left|f_{a}\right|^{-1}\left[t^{\prime}, \infty\right)$ and it preserves the stratification $\Pi_{f, \boldsymbol{\lambda}}$, i.e., we have $\Psi\left(s, \Pi_{\boldsymbol{I}, \sigma}\right) \subseteq \Pi_{\boldsymbol{I}, \sigma}$, for all $\Pi_{\boldsymbol{I}, \sigma} \in \Pi_{f, \boldsymbol{\lambda}}$ contained in $\Gamma_{t_{0}}$ and all $s \in[0,1]$.

We are now ready to conclude. However, as a warning, we note that $\Psi$ does not give a continuous retraction of $\Gamma_{t}$ onto $\Gamma_{t^{\prime}}$. The reason is that $\Gamma \mathrm{B}_{\tau}=\Gamma \mathrm{B}_{t_{0}} \cap\left|f_{a}\right|^{-1}[\tau, \infty)$ generally does not hold!

Proof of Proposition 3.2. We first show that for all $s \in[0,1]$,

$$
\Psi\left(s, \Pi_{\boldsymbol{I}, \sigma} \cap \Gamma \mathrm{B}_{t}\right) \subseteq \Pi_{\boldsymbol{I}, \sigma} \cap \Gamma_{t} \quad \text { and } \quad \Psi\left(s, \Pi_{\boldsymbol{I}, \sigma} \cap \Gamma \mathrm{B}_{t^{\prime}}\right) \subseteq \Pi_{\boldsymbol{I}, \sigma} \cap \Gamma \mathrm{B}_{t^{\prime}} .
$$

By Proposition $3.11(2)$, there are three possible cases for each of these intersections. We only focus on the third one, (iii), since the other two cases are straightforward. In this case, we have $\Pi_{\boldsymbol{I}, \sigma} \cap \Gamma_{t}=\Pi_{\boldsymbol{I}, \sigma} \cap\left\{x|| f_{a}(x) \mid \geq t\right\}$ and $\left|f_{a}\right|\left(\Pi_{\boldsymbol{I}, \sigma}\right) \subseteq\left(t_{0}, t_{1}\right)$. Thus $\Pi_{\boldsymbol{I}, \sigma} \subseteq \Omega$ and

$$
\Pi_{\boldsymbol{I}, \sigma} \cap \Gamma_{t}=\Pi_{\boldsymbol{I}, \sigma} \cap\left|f_{a}\right|^{-1}[t, \infty) .
$$

Since this is the case, again by Proposition $3.11(2)$, the same happens for $t^{\prime}$ and so

$$
\Pi_{\boldsymbol{I}, \sigma} \cap \Gamma_{t^{\prime}}=\Pi_{\boldsymbol{I}, \sigma} \cap\left|f_{a}\right|^{-1}\left[t^{\prime}, \infty\right) .
$$

Since $\Psi$ gives a deformation retract of $\Gamma_{t_{0}} \cap \alpha^{-1}\left[t, t_{1}\right)$ onto $\Gamma \mathrm{B}_{t_{0}} \cap \alpha^{-1}\left[t^{\prime}, t_{1}\right)$, it preserves the stratification $\Pi_{f, \boldsymbol{\lambda}}$, and moreover $\Psi$ gives a continuous retraction of $\Pi_{\boldsymbol{I}, \sigma} \cap\left|f_{a}\right|^{-1}[t, \infty)=$ $\Pi_{\boldsymbol{I}, \sigma} \cap\left(\Gamma_{t_{0}} \cap\left|f_{a}\right|^{-1}[t, \infty)\right)$ onto $\Pi_{\boldsymbol{I}, \sigma} \cap\left|f_{a}\right|^{-1}\left[t^{\prime}, \infty\right)$. Hence $\Psi$ must preserve $\Pi_{\boldsymbol{I}, \sigma} \cap \Gamma_{t}$ and $\Pi_{\boldsymbol{I}, \sigma} \cap \Gamma_{t^{\prime}}$ and we have shown the claim.

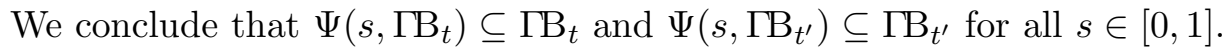

This allows us to restrict $\Psi$ to obtain continuous maps

$$
\Theta:[0,1] \times \Gamma \mathrm{B}_{t} \rightarrow \Gamma \mathrm{B}_{t}, \quad(s, x) \mapsto \Psi(s, x)
$$

and

$$
\Theta^{\prime}:[0,1] \times \Gamma \mathrm{B}_{t^{\prime}} \rightarrow \Gamma \mathrm{B}_{t^{\prime}}, \quad(s, x) \mapsto \Psi(s, x) .
$$

Let $\rho: \Gamma \mathrm{B}_{t} \rightarrow \Gamma \mathrm{B}_{t^{\prime}}$ be the continuous surjection given by

$$
x \mapsto \Psi(1, x) .
$$

By examining the three cases of Proposition 3.11(2), we see that $\rho$ is well-defined. Recall that $\iota: \Gamma \mathrm{B}_{t^{\prime}} \rightarrow \Gamma \mathrm{B}_{t}$ is the inclusion map. By construction, we have

$$
\Theta_{0}=\operatorname{id}_{\Gamma \mathrm{B}_{t}}, \quad \Theta_{1}=\rho=\iota \circ \rho, \quad \Theta_{0}^{\prime}=\operatorname{id}_{\Gamma \mathrm{B}_{t^{\prime}}} \quad \text { and } \quad \Theta_{1}^{\prime}=\rho \circ \iota .
$$

Hence, both $\left(\operatorname{id}_{\Gamma \mathrm{B}}, \iota \circ \rho\right)$ and $\left(\operatorname{id}_{\Gamma B_{t^{\prime}}}, \rho \circ \iota\right)$ are pairs of homotopic maps. Thus $\iota$ induces an homotopy equivalence as desired. 


\subsubsection{Proof of Proposition 3.11}

The way we prove this proposition is by reducing to the basic case. To do this, we write $\Phi$ in the form

$$
\Phi \equiv \bigvee_{\xi \in \Xi} \phi_{\xi}
$$

where $\Xi$ is a finite set and each $\phi_{\xi}$ is saturated. By a saturated formula over $f \in \mathcal{H}_{\boldsymbol{d}}[q]$ we mean a purely conjunctive strict formula over $f$ of the form

$$
\bigwedge_{i=1}^{q}\left(f_{i} \propto 0\right)
$$

where $\propto \in\{<,=,>\}$, i.e., a purely conjunctive strict formula over $f$ in which all components of $f$ occur. We use the word 'saturated' to emphasize that there are no polynomials left in $f$ to add to the formula.

This is possible by writing $\Phi$ in DNF and then splitting the clauses were some polynomial is missing by adding these missing polynomials with all possible sign conditions. This rewriting does not alter the sets under consideration and hence, neither does so with the validity of the statement we want to prove.

As we can take out unions in (3.2), we have

$$
\Gamma_{\tau}=\Gamma \mathrm{B}_{\delta, \varepsilon, \zeta, \tau}^{\mathcal{D}, i, a}(f, \Phi)=\bigcup_{\xi \in \Xi}\left(\Gamma_{\delta_{i}, \varepsilon_{i}, \zeta, \tau}^{\mathcal{D}, a}\left(f, \phi_{\xi}\right) \cup \bigcup_{j \neq i} \Gamma_{\delta_{j}, \varepsilon_{j}}\left(f, \phi_{\xi}\right)\right) .
$$

Hence it is enough to consider how the different strata intersect with the sets in the right hand side. This is done in Lemmas 3.12 , 3.13, and 3.14 below. We recall that we assume $\left\|f_{a}\right\|=1$ without loss of generality.

Before enunciating the lemmas, we associate to each saturated formula $\phi$ a sign vector $\operatorname{sgn}(\phi) \in\{-1,0,1\}^{q}$ given by $\operatorname{sgn}_{i}(\phi)$ equal to $-1,0$ or 1 depending on whether $\propto_{i}$ is $<,=$ or $>$, respectively. It is clear that for any $x \in \mathbb{S}^{n}, x \in \mathrm{S}(f, \phi)$ if and only if $\operatorname{sgn}(f(x))=\operatorname{sgn}(\phi)$. We endow $\{-1,0,1\}^{q}$ with a partial order: we say $\sigma \preccurlyeq \sigma^{\prime}$ iff for all $i, \sigma_{i} \neq 0$ implies $\sigma_{i}=\sigma_{i}^{\prime}$.

The first lemma deals with the $\Gamma \mathrm{B}$ blocks of the form $\Gamma_{\delta_{j}, \varepsilon_{j}}\left(f, \phi_{\xi}\right)$ with $j \neq i$, the second lemma with those of the form $\Gamma_{\delta_{i}, \varepsilon_{i}, \zeta, t_{0}}^{\mathcal{D}, a}$, and the third lemma with those of the form $\Gamma_{\delta_{\delta_{i}, \varepsilon_{i}, \zeta, \tau}^{\mathcal{D}, a}}^{\mathcal{D}}$ with $\tau \in\left(t_{0}, t_{1}\right)$. Of these, the third lemma is the most delicate one, as in this case, the $\Gamma \mathrm{B}$ blocks do not decompose as a union of strata.

Lemma 3.12. Let $\phi$ be a saturated formula over $f$, let $j \neq i$ and put $\delta:=\delta_{j}, \varepsilon:=\varepsilon_{j}$. For every $\Pi_{\boldsymbol{I}, \sigma} \in \Pi_{f, \boldsymbol{\lambda}}$ the following are equivalent:

(0I1) $\Pi_{\boldsymbol{I}, \sigma} \cap \Gamma_{\delta, \varepsilon}(f, \phi) \neq \varnothing$.

(0I2) $\Pi_{I, \sigma} \subseteq \Gamma_{\delta, \varepsilon}(f, \phi)$.

(0I3) $\operatorname{sgn}(\phi) \preccurlyeq \sigma$ and for all $l \in[q]$,

$$
\begin{cases}\left|f_{l}\right| /\left\|f_{l}\right\| \leq \varepsilon \text { on } \Pi_{\boldsymbol{I}, \sigma}, & \text { if } \operatorname{sgn}_{l}(\phi)=0 \\ \left|f_{l}\right| /\left\|f_{l}\right\| \geq \delta \text { on } \Pi_{\boldsymbol{I}, \sigma}, & \text { if } \operatorname{sgn}_{l}(\phi) \neq 0\end{cases}
$$

Proof. The chain of implications from (0I3) to (0I2) to (0I1) follows directly from the definition of $\Pi_{\boldsymbol{I}, \sigma}$. Therefore we only show that (0I1) implies (0I3).

Let $x \in \Pi_{\boldsymbol{I}, \sigma} \cap \Gamma_{\delta, \varepsilon}(f, \phi)$. For each $l \in[q]$, we distinguish three cases: 
$+)$ If $\operatorname{sgn}_{l}(\phi)=1$, then $x \in \Gamma \mathrm{B}_{\delta, \varepsilon}(f, \phi)$ implies $f_{l}(x) /\left\|f_{l}\right\| \geq \delta$. Therefore, $\sigma_{l}=\operatorname{sgn}\left(f_{l}(x)\right)=$ $1 \succcurlyeq 1=\operatorname{sgn}_{l}(\phi)$ and $\left|f_{l}\right| /\left\|f_{l}\right\| \geq \delta$ on $\Pi_{\boldsymbol{I}, \sigma}$. The latter because $\delta$ appears in in $\lambda$, and so either all $x \in \Pi_{\boldsymbol{I}, \sigma}$ satisfy $\left|f_{l}(x)\right| /\left\|f_{l}\right\| \geq \delta$ or none of them does.

-) If $\operatorname{sgn}_{l}(\phi)=-1$, the argument is analogous to that of the case $\operatorname{sgn}_{l}(\phi)=1$.

$0)$ If $\operatorname{sgn}_{l}(\phi)=0$, then $x \in \Gamma \mathrm{B}_{\delta, \varepsilon}(f, \phi)$ implies $\left|f_{l}(x)\right| /\left\|f_{l}\right\| \leq \varepsilon$. This, in turn, implies $\left|f_{l}\right| /\left\|f_{l}\right\| \leq \varepsilon$ on $\Pi_{\boldsymbol{I}, \sigma}$, since $\varepsilon$ appears in $\lambda$, and so either all $x \in \Pi_{\boldsymbol{I}, \sigma}$ satisfy this or none does. Also $0 \preccurlyeq 0,+1,-1$, and $\operatorname{sos} \operatorname{sgn}\left(f_{l}(x)\right) \preccurlyeq \sigma_{l}$.

Lemma 3.13. Let $\phi$ be a saturated formula over $f$. For every $\Pi_{\boldsymbol{I}, \sigma} \in \Pi_{f, \boldsymbol{\lambda}}$, the following are equivalent:

(1I1) $\Pi_{\boldsymbol{I}, \sigma} \cap \Gamma_{\delta_{i}, \varepsilon_{i}, \zeta, t_{0}}^{\mathcal{D}, a}(f, \phi) \neq \varnothing$.

(1I2) $\Pi_{\boldsymbol{I}, \sigma} \subseteq \Gamma_{\delta_{i}, \varepsilon_{i}, \zeta, t_{0}}^{\mathcal{D}, a}(f, \phi)$.

(1I3) $\operatorname{sgn}(\phi) \preccurlyeq \sigma$ and, for all $l \in[q]$,

$$
\begin{cases}\left|f_{l}\right| /\left\|f_{l}\right\| \leq \varepsilon_{i} \text { on } \Pi_{\boldsymbol{I}, \sigma}, & \text { if } \operatorname{sgn}_{l}(\phi)=0, \\ \left|f_{l}\right| /\left\|f_{l}\right\| \geq \delta_{i} \text { on } \Pi_{\boldsymbol{I}, \sigma}, & \text { if } \operatorname{sgn}_{l}(\phi) \neq 0 \text { and } l>a, \\ \left|f_{l}\right| /\left\|f_{l}\right\| \geq t_{0} \text { on } \Pi_{\boldsymbol{I}, \sigma}, & \text { if } \operatorname{sgn}_{l}(\phi) \neq 0 \text { and } l=a, \\ \left|f_{l}\right| /\left\|f_{l}\right\| \geq \zeta \text { on } \Pi_{\boldsymbol{I}, \sigma}, & \text { if } \operatorname{sgn}_{l}(\phi) \neq 0 \text { and } l<a .\end{cases}
$$

Proof. The proof is analogous to that of Lemma 3.12, but longer as we must now divide into cases depending not only on $\operatorname{sgn}_{l}(\phi)$ but also on whether $l>a, l=a$ or $l<a$.

Lemma 3.14. Let $\phi$ be a saturated formula over $f$ and $s \in\left(t_{0}, t_{1}\right)$. For every $\Pi_{\boldsymbol{I}, \sigma} \in \Pi_{f, \boldsymbol{\lambda}}$ the following are equivalent:

$$
\Pi_{\boldsymbol{I}, \sigma} \cap \Gamma \mathrm{B}_{\delta_{i}, \varepsilon_{i}, \zeta, s}^{\mathcal{D}, a}(f, \phi) \neq \varnothing .
$$

(2I2) $\operatorname{sgn}(\phi) \preccurlyeq \sigma$ and for all $l \in[q]$,

$$
\begin{cases}\left|f_{l}\right| /\left\|f_{l}\right\| \leq \varepsilon_{i} \text { on } \Pi_{\boldsymbol{I}, \sigma}, & \text { if } \operatorname{sgn}_{l}(\phi)=0, \\ \left|f_{l}\right| /\left\|f_{l}\right\| \geq \delta_{i} \text { on } \Pi_{\boldsymbol{I}, \sigma}, & \text { if } \operatorname{sgn}_{l}(\phi) \neq 0 \text { and } l>a, \\ \left|f_{l}\right| /\left\|f_{l}\right\|>t_{0} \text { on } \Pi_{\boldsymbol{I}, \sigma}, & \text { if } \operatorname{sgn}_{l}(\phi) \neq 0 \text { and } l=a, \\ \left|f_{l}\right| /\left\|f_{l}\right\| \geq \zeta \text { on } \Pi_{\boldsymbol{I}, \sigma}, & \text { if } \operatorname{sgn}_{l}(\phi) \neq 0 \text { and } l<a .\end{cases}
$$

Additionally, if any of the two claims above holds,

$$
\Pi_{\boldsymbol{I}, \sigma} \cap \Gamma \mathrm{B}_{\delta_{i}, \varepsilon_{i}, \zeta, \tau}^{\mathcal{D}, a}(f, \phi)= \begin{cases}\Pi_{\boldsymbol{I}, \sigma} \cap\left\{x \in \mathbb{S}^{n}|| f_{a}(x) \mid \geq \tau\right\}, & \text { if }\left|f_{a}\right|\left(t_{0}, t_{1}\right) \subseteq \Pi_{\boldsymbol{I}, \sigma}, \\ \Pi_{\boldsymbol{I}, \sigma}, & \text { otherwise. }\end{cases}
$$

Proof. The implication from (2I1) to (2I2) is shown in a similar way as those from (0I1) to (0I3) in Lemma 3.12 and from (1I1) to (1I3) in Lemma 3.13. We next prove the reverse implication. 
Assume then that (2I2) holds. From the conditions there and the definition of both $\Pi_{\boldsymbol{I}, \sigma}$ and $\Gamma_{\delta_{i}, \varepsilon_{i}, \zeta, s}^{\mathcal{D}, a}(f, \phi)$, it follows that

$$
\Pi_{\boldsymbol{I}, \sigma} \cap \Gamma_{\delta_{i}, \varepsilon_{i}, \zeta, s}^{1, a}(f, \phi)=\Pi_{\boldsymbol{I}, \sigma} \cap\left\{x \in \mathbb{S}^{n}|| f_{a}(x) \mid \geq s\right\} .
$$

We next divide in cases depending on whether $\Pi_{\boldsymbol{I}, \sigma} \subseteq\left|f_{a}\right|^{-1}\left(t_{0}, t_{1}\right)$ or not.

$\nsubseteq)$ If $\left|f_{a}\right|\left(t_{0}, t_{1}\right) \nsubseteq \Pi_{\boldsymbol{I}, \sigma}$, then $\left|f_{a}\right| \geq t_{1}$ on $\Pi_{\boldsymbol{I}, \sigma}$, by (2I2), since $t_{1}$ is the next value in $\lambda_{a}$. This shows that

$$
\Pi_{\boldsymbol{I}, \sigma} \cap\left\{x \in \mathbb{S}^{n}|| f_{a}(x) \mid \geq s\right\}=\Pi_{\boldsymbol{I}, \sigma} .
$$

As $\Pi_{I, \sigma}$ is non-empty, (2I1) follows from (3.10) and (3.11).

$\subseteq)$ If, instead, $\left|f_{a}\right|\left(t_{0}, t_{1}\right) \subseteq \Pi_{\boldsymbol{I}, \sigma}$ then, by Theorem 3.9(2), the map

$$
\Pi_{\boldsymbol{I}, \sigma} \rightarrow\left(t_{0}, t_{1}\right), x \mapsto\left|f_{a}(x)\right| \|
$$

is surjective. Hence $\Pi_{\boldsymbol{I}, \sigma} \cap\left\{x \in \mathbb{S}^{n}|| f_{a}(x) \mid \geq \tau\right\}$ is non-empty and (2I1) also follows in this case.

We have proved (3.9) in passing.

Now we finish the proof of Proposition 3.11 with the help of the above three lemmas.

Proof of Proposition 3.11. Part (1) follows directly from Lemmas 3.12 and 3.13 since these lemmas guarantee that each set in the right-hand side of (3.8) is a union of strata.

We now show part (2). Consider the intersections of $\Pi_{\boldsymbol{I}, \sigma}$ with the decomposition (3.8) for $\Gamma_{\tau}$ and $\Gamma_{t_{0}}$.

If for some $j \neq i$ and $\xi \in \Xi$ we have $\Pi_{\boldsymbol{I}, \sigma} \cap \Gamma_{\delta_{j}, \varepsilon_{j}}\left(f, \phi_{\xi}\right) \neq \varnothing$, then this intersection equals $\Pi_{\boldsymbol{I}, \sigma}$ by Lemma 3.12 and all the claims of (2) hold trivially since $\Pi_{\boldsymbol{I}, \sigma} \cap \Gamma \mathrm{B}_{\delta_{j}, \varepsilon_{j}}\left(f, \phi_{\xi}\right)$ does not depend on the value of $\tau$.

Assume instead that for all $j \neq i$ and $\xi \in \Xi$ we have $\Pi_{\boldsymbol{I}, \sigma} \cap \Gamma \mathrm{B}_{\delta_{j}, \varepsilon_{j}}\left(f, \phi_{\xi}\right)=\varnothing$. Then

$$
\Pi_{\boldsymbol{I}, \sigma} \cap \Gamma_{\tau}=\bigcup_{\xi \in \Xi} \Pi_{\boldsymbol{I}, \sigma} \cap \Gamma \mathrm{B}_{\delta_{i}, \varepsilon_{i}, \zeta, \tau}^{\mathcal{D}, a}\left(f, \phi_{\xi}\right)
$$

and

$$
\Pi_{\boldsymbol{I}, \sigma} \cap \Gamma \mathrm{B}_{t_{0}}=\bigcup_{\xi \in \Xi} \Pi_{\boldsymbol{I}, \sigma} \cap \Gamma \mathrm{B}_{\delta_{i}, \varepsilon_{i}, \zeta, t_{0}}^{\mathcal{D}, a}\left(f, \phi_{\xi}\right) .
$$

By hypothesis on $\Pi_{\boldsymbol{I}, \sigma}$, we have $\Pi_{\boldsymbol{I}, \sigma} \cap \Gamma_{t_{0}}=\Pi_{\boldsymbol{I}, \sigma} \neq \varnothing$ which implies that there exists $\xi \in \Xi$ such that $\Pi_{\boldsymbol{I}, \sigma} \cap \Gamma_{\delta_{i}, \varepsilon_{i}, \zeta, t_{0}}^{\mathcal{D}, a}\left(f, \phi_{\xi}\right) \neq \varnothing$. Lemma 3.13 then ensures that the conditions in (1I3) hold true. But these conditions are the same as those in Lemma 3.14(212) except for $l=a$, where the inequality is strict in the latter and lax in the proper. This means that $\Pi_{\boldsymbol{I}, \sigma} \cap \Gamma_{\boldsymbol{\delta}, \varepsilon, \zeta, \tau}^{\mathcal{D}, i, a}(f, \Phi)=\varnothing$ if and only if $\left|f_{a}\right|=t_{0}$ on $\Pi_{\boldsymbol{I}, \sigma}$. Furthermore, this latter condition is independent of the particular value of $\tau$. If it holds for $\tau$, then it holds for $\tau^{\prime}$ and viceversa. This proves the first claim of (2).

Arguing as above, we have that $\Pi_{\boldsymbol{I}, \sigma} \cap \Gamma_{\boldsymbol{\delta}, \varepsilon, \zeta, s}^{\mathcal{D}, i, a}(f, \Phi)=\Pi_{\boldsymbol{I}, \sigma}$ if and only if $\left|f_{a}\right| \geq t_{1}$ on $\Pi_{\boldsymbol{I}, \sigma}$. As this does not depend on the value of $\tau$, we get the second claim of (2).

The third claim of (2) follows directly from the last statement of Lemma 3.14 , 


\section{Sampling theory for Gabrielov-Vorobjov approximations}

In this section we prove the remaining stepping stones we introduced in the overview (Section 2) namely, Theorems 2.13, 2.14, and 2.15, The core of these proofs relies on the fact that the $\gamma$ invariant of Smale (see 4.2 below) at points in $\mathbb{S}^{n}$ does not change much when we replace the homogeneous polynomials in $f$ by the perturbations $\bar{f}$. Otherwise, these three results are extensions of similar results in [2] and most of the arguments we use are exactly those we used there to prove the simpler versions. We will therefore be concise and, in most places in what follows, limit our exposition to the general lines, omitting the details.

\subsection{Semialgebraic sets from $(f, t)$ and related results}

Recall from Definition 2.11 the notion of a lax formula over $(f, t)$ and of its associated spherical sets $\mathrm{S}(f, t, \Phi)$. We define now algebraic neighborhoods of the sets $\mathrm{S}(f, t, \Phi)$ by relaxing the inequalities.

Definition 4.1. The algebraic neighborhood $\mathrm{S}_{r}(f, t, \Phi)$ of $\mathrm{S}(f, t, \Phi)$ with tolerance $r>0$ is the spherical semialgebraic set defined by replacing the atoms $f_{i} \geq t_{j}\left\|f_{i}\right\|$ by $f_{i} \geq$ $\left(t_{j}-r\right)\left\|f_{i}\right\|$ and the atoms $f_{i} \leq t_{j}\left\|f_{i}\right\|$ by $f_{i} \leq\left(t_{j}+r\right)\left\|f_{i}\right\|$. By using strict inequalities, we define the open algebraic neighborhood $\mathrm{S}_{r}^{\circ}(f, t, \Phi)$ of $\mathrm{S}(f, t, \Phi)$ with tolerance $r$.

Remark 4.2. We use the term "algebraic neighborhood" instead of the arguably more correct term "semialgebraic neighborhood" for the sake of conciseness and for consistency with [2].

The following three quantitative results about algebraic neighborhoods generalize, respectively, Proposition 4.17 and Theorem 4.19 in [1], and Theorem 2.7 in [2]. In contrast with these result, the separation $\delta(t):=\inf _{i \neq j}\left|t_{i}-t_{j}\right|$ of $t$ enters here as a new parameter. Note that the sequence $t$ defined (2.7) has the separation

$$
\delta(t)=\left(15(2 m+1) D^{2} \mathrm{~K}^{2}\right)^{-1} .
$$

Similarly to (2.9), we denote by $\mathcal{U}_{\mathbb{S}}(X, r)$ the open (spherical) $r$-neighborhood of a subset $X$ of $\mathbb{S}^{n}$, which is defined with respect to angular distance. Clearly, $\mathcal{U}_{\mathbb{S}}(X, r) \subseteq$ $\mathcal{U}(X, r)$.

Proposition 4.3. Let $f \in \mathcal{H}_{\boldsymbol{d}}[q], t \in \mathbb{R}^{e}$ and $r>0$. Then, for every lax formula $\Phi$ over $(f, t)$, we have

$$
\mathcal{U}_{\mathbb{S}}(\mathrm{S}(f, t, \Phi), r) \subseteq \mathrm{S}_{D^{1 / 2} r}^{\circ}(f, t, \Phi) .
$$

Proposition 4.4. Let $f \in \mathcal{H}_{\boldsymbol{d}}[q]$ and $T, r>0$ be such that $13 D^{3 / 2} \bar{\kappa}(f)^{2}(r+T)<1$. Then, for all $t \in(-T, T)^{e}$ satisfying $\delta(t)>2 r$ and every purely conjunctive lax formula $\phi$ over $(f, t)$, we have

$$
\mathrm{S}_{r}^{\circ}(f, t, \phi) \subseteq \mathcal{U}_{\mathbb{S}}(\mathrm{S}(f, t, \phi), 3 \bar{\kappa}(f) r) .
$$

Theorem 4.5 (Generalized Quantitative Durfee Theorem). Let $f \in \mathcal{H}_{\boldsymbol{d}}[q]$ and $T, r>0$ be such that $\sqrt{2} \bar{\kappa}(f)(r+T)<1$. Then, for all $t \in(-T, T)^{e}$ satisfying $\delta(t)>2 r$ and every purely conjunctive lax formula $\phi$ over $(f, t)$, the inclusions in

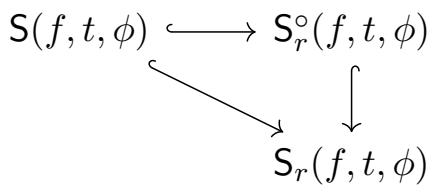


are homotopy equivalences.

These three results are proved in the same manner as their corresponding results in [1] and [2]. We briefly describe how the differences in the statements occur.

1. The condition $\sqrt{2} \bar{\kappa}(f) r<1$ becomes $\sqrt{2} \bar{\kappa}(f)(r+T)<1$. In the case $t=0$, this condition guaranteed that when $\left\|f^{L}(x)\right\| /\left\|f^{L}\right\|<1 /(\sqrt{2} \bar{\kappa}(f))$ for a suitably chosen index set $L \subseteq[q]$, one could apply [2, Proposition 3.6] to deduce that $\mathrm{D}_{x} f^{L}$ is surjective. For such $L$, we had $\left\|f^{L}(x)\right\| /\left\|f^{L}\right\| \leq r$ since the point $x$ was lying in $\mathrm{S}_{r}(f, \phi) \backslash \mathrm{S}(f, \phi)$. In our current setting, we will have $\left\|f^{L}(x)\right\| /\left\|f^{L}\right\| \leq r+T$ and so we have to replace $r$ by $r+T$ in the bound in the hypotheses to make the argument work.

2. The addition of the condition $\delta(t)>2 r$. This condition guarantees that the set $\mathrm{S}_{r}\left(f, t,\left(f_{i} \geq t_{j}\left\|f_{i}\right\|\right) \wedge\left(f_{i} \leq t_{j^{\prime}}\left\|f_{i}\right\|\right) \wedge \psi\right)$ is empty whenever $t_{j^{\prime}}<t_{j}$. This phenomenon is the only obstruction to assume without loss of generality that the formula $\phi$ in the statements of Proposition 4.4 and Theorem 4.5 is of the form

$$
\left.\bigwedge_{i \in I}\left(f_{i} \leq\left\|f_{i}\right\| t_{\alpha(i)}\right) \wedge \bigwedge_{j \in J}\left(f_{j} \geq\left\|f_{j}\right\| t_{\alpha(j)}\right) \wedge \bigwedge_{k \in K}\left(f_{k} \leq\left\|f_{k}\right\| t_{\beta(k)}\right) \wedge\left(f_{k} \geq\left\|f_{k}\right\| t_{\gamma(k)}\right)\right)
$$

for some disjoint sets $I, J, K \subseteq\{1, \ldots, q\}$, and maps $\alpha: I \cup J \rightarrow\{1, \ldots, e\}, \beta: K \rightarrow$ $\{1, \ldots, e\}$ and $\gamma: K \rightarrow\{1, \ldots, e\}$ such that for $k \in K, t_{\beta(k)} \geq t_{\gamma(k)}$.

A complete proof reworking out all the details can be found in [17, Ch. 2]. We note, however, that the proofs in [17] don't follow exactly the lines of the proofs in [1, 2], although they use the same underlying fundamental ideas.

\subsection{Proof of Theorem 2.13}

Towards the proof of this result we recall the definition of the gamma invariant defined by Smale [16] (see also [15, 3]) in both its affine and projective versions.

Let $n \geq m$. For a map $G: \mathbb{R}^{n+1} \rightarrow \mathbb{R}^{m}$ and a point $x \in \mathbb{S}^{n}$, we let $\overline{\mathrm{D}}_{x} G: \mathbb{R}^{n+1} \rightarrow \mathbb{R}^{m}$ be the derivative at $x$ of $G$ viewed as a map on the Euclidean space and $\mathrm{D}_{x} G: \mathrm{T}_{x} \mathbb{S}^{n} \rightarrow \mathbb{R}^{m}$ denote the derivative at $x$ of $G$ as a map on the sphere. Smale's (Euclidean) gamma of $G: \mathbb{R}^{n+1} \rightarrow \mathbb{R}^{m}$ at $x \in \mathbb{S}^{n}$ is the number given by

$$
\bar{\gamma}(G, x):= \begin{cases}\sup _{k \geq 2}\left\|\overline{\mathrm{D}}_{x} G^{\dagger} \frac{1}{k !} \overline{\mathrm{D}}_{x}^{k} G\right\|, & \text { if } \overline{\mathrm{D}}_{x} G^{\dagger} \text { surjective } \\ \infty, & \text { otherwise. }\end{cases}
$$

The projective Smale's gamma $\gamma(G, x)$ of $G$ at $x$ is defined similarly, with $\overline{\mathrm{D}}_{x} G$ replaced by $\mathrm{D}_{x} G$.

We recall from [2] the condition number of $f \in \mathcal{H}_{\boldsymbol{d}}[m]$ at $x$, defined by $\mu(f, x):=$ $\|f\|\left\|D_{x} f^{\dagger} \Delta\right\|$, where $\Delta:=\operatorname{diag}\left(\sqrt{d_{1}}, \ldots, \sqrt{d_{m}}\right)$. (Note that this quantity was denoted $\mu_{\text {proj }}(f, x)$ in [1].) We also recall the Higher Derivative Estimate, $\gamma(f, x) \leq \frac{1}{2} D^{3 / 2} \mu(f, x)$, from [3, Theorem 16.1]. (This reference only covers the case $m=n$, but the proof given there immediately extends to $n \geq m$ ).

With these definitions, one proves the following proposition. 
Proposition 4.6. Let $f \in \mathcal{H}_{\boldsymbol{d}}[m]$, with $n \geq m$, and $x \in \mathbb{S}^{n}$. Define $f_{\mathbb{S}}:=\left(f, \sum_{i=0}^{n} X_{i}^{2}-1\right)$. Then

$$
2 \bar{\gamma}\left(f_{\mathbb{S}}, x\right) \leq D^{\frac{3}{2}} \mu(f, x)+D^{\frac{1}{2}} \mu(f, x) \frac{\|f(x)\|}{\|f\|}+1 .
$$

Proof. By direct computation,

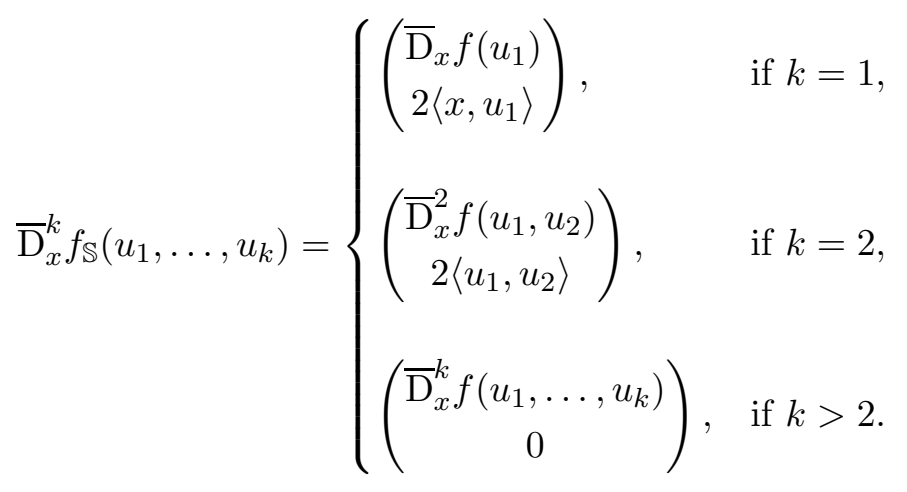

Using this equality for $k=1$ we deduce that $\operatorname{ker} \overline{\mathrm{D}}_{x} f_{\mathbb{S}}=\mathrm{T}_{x} \mathbb{S}^{n} \cap \operatorname{ker} \overline{\mathrm{D}}_{x} f$. Let $V$ be the orthogonal complement of ker $\overline{\mathrm{D}}_{x} f$ in $\mathrm{T}_{x} \mathbb{S}^{n}$. Then $\left(\operatorname{ker} \overline{\mathrm{D}}_{x} f_{\mathbb{S}}\right)^{\perp}=V+\mathbb{R} x$ and, for all $\lambda \in \mathbb{R}$,

$$
\overline{\mathrm{D}}_{x} f_{\mathbb{S}}(v+\lambda x)=\left(\begin{array}{c}
\mathrm{D}_{x} f(v)+\lambda \Delta^{2} f(x) \\
2 \lambda
\end{array}\right)
$$

where, we recall, $\Delta:=\operatorname{diag}\left(\sqrt{d_{1}}, \ldots, \sqrt{d_{m}}\right)$ and $\overline{\mathrm{D}}_{x} f(x)=\Delta^{2} f(x)$ follows from Euler's identity for homogeneous functions.

By explicitly inverting the map in (4.4), we obtain

$$
\left(\overline{\mathrm{D}}_{x} f_{\mathbb{S}}\right)^{\dagger}\left(\begin{array}{c}
w \\
t
\end{array}\right)=\mathrm{D}_{x} f^{\dagger}\left(w-\frac{t}{2} \Delta^{2} f(x)\right)+\frac{t}{2} x
$$

Thus

$$
\left(\overline{\mathrm{D}}_{x} f_{\mathbb{S}}\right)^{\dagger} \frac{\overline{\mathrm{D}}_{x}^{k} f_{\mathbb{S}}}{k !}\left(u_{1}, \ldots, u_{k}\right)= \begin{cases}\mathrm{D}_{x} f^{\dagger} \frac{\overline{\mathrm{D}}_{x}^{2} f}{2}\left(u_{1}, u_{2}\right)-\frac{\left\langle u_{1}, u_{2}\right\rangle}{2} \mathrm{D}_{x} f^{\dagger} \Delta^{2} f(x)+\frac{\left\langle u_{1}, u_{2}\right\rangle}{2} x, & \text { if } k=2, \\ \mathrm{D}_{x} f^{\dagger} \frac{\overline{\mathrm{D}}_{x}^{k} f}{k !}\left(u_{1}, \ldots, u_{k}\right), & \text { if } k>2 .\end{cases}
$$

Applying the triangular inequality in (4.2), we obtain

$$
\bar{\gamma}\left(f_{\mathbb{S}}, x\right) \leq \gamma(f, x)+\frac{1}{2}\left\|\mathrm{D}_{x} f^{\dagger} \Delta^{2} f(x)\right\|+\frac{1}{2},
$$

which implies

$$
2 \bar{\gamma}\left(f_{\mathbb{S}}, x\right) \leq D^{\frac{3}{2}} \mu(f, x)+D^{\frac{1}{2}} \mu(f, x) \frac{\|f(x)\|}{\|f\|}+1
$$

where the first term in the right-hand side follows from the Higher Derivative Estimate and the second from the relations

$$
\left\|\mathrm{D}_{x} f^{\dagger} \Delta^{2} f(x)\right\| \leq\left\|\mathrm{D}_{x} f^{\dagger} \Delta\right\|\|\Delta\|\|f(x)\|=\|f\|\left\|\mathrm{D}_{x} f^{\dagger} \Delta\right\| D^{\frac{1}{2}} \frac{\|f(x)\|}{\|f\|}
$$

and the definition of $\mu$. This finishes the proof. 
Proof of Theorem 2.13. We refer to [1] for the definition of the reach $\tau(X)$ of a subset $X$ of Euclidean space, and its local reach $\tau(X, p)$ at $p \in X$. By [1, Theorem 2.8] (a variant of the Niyogi-Smale-Weinberger Theorem [12]), it is sufficient to prove that

$$
\frac{1}{48 D^{\frac{3}{2}} \bar{\kappa}(f)}<\frac{1}{2} \tau(\mathrm{S}(f, t, \phi))
$$

Without loss of generality, we can write $\phi$ as $\bigwedge_{i \in I}\left(f_{i} \leq\left\|f_{i}\right\| t_{\beta(i)}\right) \wedge \bigwedge_{j \in J}\left(f_{j} \geq\left\|f_{j}\right\| t_{\beta^{\prime}(j)}\right)$ for some $I, J \subseteq[q]$ and some maps $\beta: I \rightarrow[e]$ and $\beta^{\prime}: J \rightarrow[e]$.

For $L \subseteq I$ with $|L| \leq n$ and $\alpha: L \rightarrow[e]$ we consider the (Euclidean) zero-set

$$
W_{L, \alpha}:=\bigcap_{i \in L} \mathrm{~S}\left(f_{i}-\left\|f_{i}\right\| t_{\alpha(i)}=0\right) .
$$

of $\left(f^{L}-\delta^{L, \alpha}, \sum_{i=0}^{n} X_{i}^{2}-1\right)$, where we have put $\delta^{L, \alpha}:=\left(\left\|f_{i}\right\| t_{\alpha(i)}\right)_{i \in L} \in \mathbb{R}^{L}$. By [1, Corollary 2.6], we have

$$
\tau(\mathrm{S}(f, t, \phi)) \geq \min \left\{\tau\left(W_{L, \alpha}\right)|L \subseteq I,| L \mid \leq n, \alpha: L \rightarrow[e]\right\},
$$

since the boundary of both sets $\mathbf{S}\left(f_{i}-\left\|f_{i}\right\| t_{j} \geq 0\right)$ and $\mathbf{S}\left(f_{i}-\left\|f_{i}\right\| t_{j} \leq 0\right)$ equals $\mathbf{S}\left(f_{i}-\right.$ $\left.\left\|f_{i}\right\| t_{j}=0\right)$. The latter follows by the implicit function theorem, which can be applied due to [2, Proposition 3.6] and $\sqrt{2} \bar{\kappa}(f) T<1$. Moreover, by [1, Theorem 2.11], we have (recall the definition of $\bar{\gamma}$ in (4.2) $)$

$$
\tau\left(W_{L, \alpha}, p\right) \geq \frac{1}{14 \bar{\gamma}\left(\left(f^{L}-\delta^{L, \alpha}, \sum_{i=0}^{n} X_{i}^{2}-1\right), p\right)} .
$$

On the one hand, we have that

$$
\frac{\left\|f^{L}(p)\right\|}{\left\|f^{L}\right\|} \leq \max _{i \in L} \frac{\left|f_{i}(p)\right|}{\left\|f_{i}\right\|}<T<\frac{1}{\sqrt{2} \bar{\kappa}(f)}
$$

where for the second inequality, we have used that $p \in W_{L}$.

On the other hand, we have $\mu\left(f^{L}, p\right) \leq \sqrt{2} \kappa\left(f^{L}\right)$, by [2, Proposition 3.6].

Putting these together, we deduce with Proposition 4.6 that

$$
\begin{aligned}
2 \bar{\gamma}\left(\left(f^{L}-\delta^{L, \alpha}, \sum_{i=0}^{n} X_{i}^{2}-1\right), p\right) & <D^{\frac{3}{2}} \mu\left(f^{L}, p\right)+D^{\frac{1}{2}} \mu\left(f^{L}, p\right) T+1 \\
& <\sqrt{2} D^{\frac{3}{2}} \kappa\left(f^{L}\right)+D^{\frac{1}{2}}+1 \leq(\sqrt{2}+2) D^{\frac{3}{2}} \kappa\left(f^{L}\right),
\end{aligned}
$$

where we used $\kappa\left(f^{L}\right) \geq 1$ for the last inequality. Combining (4.6) and (4.7) the claim (4.5) follows and the proof is complete.

\subsection{Proof of Theorem 2.14}

Theorem 2.14 follows from the following more general result.

Theorem 4.7. Let $f \in \mathcal{H}_{\boldsymbol{d}}[q]$ and $T>0$ be such that $2 \bar{\kappa}(f) T<1$, and $\varepsilon>0$. Let $t \in(-T, T)^{e}$. Moreover, for $i \in[q]$ and $j \in[e]$, let $\mathcal{X}_{i, j}^{\leq}, \mathcal{X}_{i, j}^{\geq} \subseteq \mathbb{S}^{n}$ be finite subsets such that for all purely conjunctive lax formulas $\phi$ over $(f, t)$, we have

$3 d_{H}\left(\phi\left(\mathcal{X}_{i, j}^{\propto} \mid i \in[q], j \in[e], \propto \in\{\leq, \geq\}\right), \mathrm{S}(f, t, \phi)\right)<\varepsilon<\min \left\{\frac{1}{48 D^{3 / 2} \bar{\kappa}(f)}, \frac{\delta(t)}{12 D^{1 / 2}}\right\}$. 
Then, for all lax formulas $\Psi$ over $(f, t)$, the set $\mathrm{S}(f, t, \Psi)$ and the simplicial complex

$$
\Psi\left(\check{\mathrm{C}}_{\varepsilon}\left(\mathcal{X}_{i, j}^{\propto}\right) \mid i \in[q], j \in[e], \propto \in\{\leq, \geq\}\right)
$$

have the same homology.

Proof. The proof follows the same lines as that of the Homology Witness Theorem [2, Theorem 2.4], now relying on Theorem 4.5, Theorem 2.13, and Proposition 4.3 instead on Theorem 2.7, Theorem 2.3, and inequality (2.4) in [2, respectively.

The main difficulty in the proof is to find some $r$ that satisfies certain inequalities so that the three results above can be applied. Because of the new bounds in the extended results, we now need to find $r>0$ such that both $\sqrt{2} \bar{\kappa}(f)(r+T)<1$ and $r<\frac{1}{2} \delta(t)$ hold (hypothesis of Theorem 4.5) and $6 D^{\frac{1}{2}} \varepsilon \leq r$ (to conveniently use the bounds in Proposition 4.3). Such $r$ can be found, provided

$$
6 D^{\frac{1}{2}} \varepsilon<\min \left\{\frac{1}{\sqrt{2} \bar{\kappa}(f)}-T, \frac{1}{2} \delta(t)\right\} .
$$

The assumption on $\varepsilon$ implies that

$$
6 D^{\frac{1}{2}} \varepsilon<\min \left\{\frac{1}{8 D \bar{\kappa}(f)}, \frac{1}{2} \delta(t)\right\}
$$

Now we note that

$$
\min \left\{\frac{1}{8 D \bar{\kappa}(f)}, \frac{1}{2} \delta(t)\right\}<\min \left\{\frac{1}{\sqrt{2} \bar{\kappa}(f)}-T, \frac{1}{2} \delta(t)\right\}
$$

since

$$
T<\frac{8 D-\sqrt{2}}{8 \sqrt{2} D} \frac{1}{\bar{\kappa}(f)}
$$

which in turn is guaranteed by the assumption $2 \bar{\kappa}(f) T<1$ and $D \geq 1$.

Proof of Theorem 2.14. Let $e=4 m$ and $t$ be given by (2.7) and put $T:=(2 m+1) \delta(t)$. By Proposition 2.2 and (4.1), we have

$$
\sqrt{2} \bar{\kappa}(f) T=\sqrt{2} \bar{\kappa}(f) \frac{1}{15 D^{2} \mathrm{~K}^{2}}<1 .
$$

Also, Proposition 2.2 and (4.1) imply that

$$
\frac{1}{48 D^{3 / 2} \bar{\kappa}(f)}>\frac{1}{180(2 m+1) D^{5 / 2} \mathrm{~K}^{2}}=\frac{\delta(t)}{12 D^{1 / 2}} .
$$

Hence, all the hypotheses of Theorem 4.7 are satisfied and the conclusion follows from applying this result to formulas of the form $\bar{\Phi}$ over $(f, t)$ constructed from strict formulas $\Phi$ over $f$. 


\subsection{Proof of Theorem 2.15}

Again, Theorem 2.15 is an immediate consequence of the following more general result.

Theorem 4.8. Let $f \in \mathcal{H}_{\boldsymbol{d}}[q]$ and $T, r>0$ be such that $13 D^{2} \bar{\kappa}(f)^{2}(r+T)<1$. Assume $t \in(-T, T)^{e}$ satisfies $\delta(t)>2 D^{\frac{1}{2}} r$ and let $\Phi$ be a strict formula over $(f, t)$. Then for every finite set $\mathcal{G} \subseteq \mathbb{S}^{n}$ such that $d_{H}\left(\mathcal{G}, \mathbb{S}^{n}\right)<r$, we have

$$
d_{H}\left(\mathrm{~S}_{D^{\frac{1}{2}} r}^{\circ}(f, t, \Phi) \cap \mathcal{G}, \mathrm{S}(f, t, \Phi)\right)<3 D^{\frac{1}{2}} \bar{\kappa}(f) r .
$$

Proof. The proof is the same as that of [2, Theorem 6.5], using now Propositions 4.4 and 4.3 in the place of, respectively, Proposition 2.6 and inequality (2.4) in [2].

Proof of Theorem 2.15. Let $e=4 m, t$ be given by (2.7) and $T:=\left(2 m+1-\frac{1}{108 D \mathrm{~K}}\right) \delta(t)$. Using that $\delta(t)=\left(15(2 m+1) D^{2} \mathrm{~K}^{2}\right)^{-1}$, we get

$$
r_{\ell} \leq \frac{\rho}{108 D \mathrm{~K}} \delta(t) \leq \frac{1}{108 D \mathrm{~K}} \delta(t)
$$

We verify that the hypothesis of Theorem 4.8 is satisfied for $r=r_{\ell}$. On the one hand, using (4.8) and the definition of $T$, we get

$$
13 D^{2} \bar{\kappa}(f)^{2}\left(r_{\ell}+T\right) \leq 13 D^{2} \bar{\kappa}(f)^{2}(2 m+1) \delta(t)=\frac{13 \bar{\kappa}(f)^{2}}{15 D^{2} \mathrm{~K}^{2}}<1
$$

where the last inequality follows from Proposition [2.2. On the other hand, using (4.8) again, we have

$$
2 D^{\frac{1}{2}} r_{\ell}<\delta(t) .
$$

Finally, using (4.8) one more time, the result follows from Theorem 4.8, because

$$
3 \cdot 3 D^{\frac{1}{2}} \bar{\kappa}(f) r_{l} \leq \frac{9 \bar{\kappa}(f) \rho}{108 D^{1 / 2} \mathrm{~K}} \delta(t) \leq \frac{\rho}{12 D^{1 / 2}} \delta(t),
$$

where the last inequality follows from Proposition 2.2,

\section{Concluding remarks}

Cylindrical Algebraic Decomposition (CAD) [4, 18] as refined in [14] may be used to compute the homology of semialgebraic sets with a worst-case complexity bounded by $(q D)^{2^{\mathcal{O}(n)}}$. One can combine CAD with Algorithm Homology (by running both of them "in parallel") to obtain a numeric-symbolic algorithm, call it Hybrid, which enjoys (under infinite precision) the virtues of both CAD and Homology. It has a weak singly exponential cost (thus exponentially accelerating the cost of CAD) with a doubly exponential worstcase cost (thus overcoming the major shortcoming of Homology, the fact that it does not solve ill-posed data and that it takes too long for data close to ill-posed).

(1) The complexity bounds for Homology (or Hybrid), under infinite precision assumption, have a different nature than those for CAD. Algorithm Homology has an input-dependent complexity bound and this bound is probabilistic, while the bound for CAD is inputindependent and deterministic. This means that in order to make a fair comparison it would be helpful to have an answer to the following question. 
Question 5.1. Is the worst-case complexity bound $(q D)^{2^{\mathcal{O}(n)}}$ of CAD attained for almost all inputs? Or can it be improved for a random input as in Theorem 1.1]

Ignoring this issue, and assuming that no better bounds are possible, we can say that Algorithm Homology is faster than CAD with high probability. In the general case, Homology is faster than CAD (with probability at least $1-(n q D)^{-n}$ ) because

$$
\operatorname{size}(\Phi) q^{\mathcal{O}(n)}(n D)^{\mathcal{O}\left(n^{3}\right)} \leq(q D)^{2^{\mathcal{O}(n)}} .
$$

In the particular case of families of inputs for which the degree $D \geq 2$ is bounded and the number $q$ of polynomials is moderate, we have $N=n^{\mathcal{O}(1)}$. In this case, Hybrid is faster than CAD (with probability at least $1-2^{-\operatorname{size}(p, \Phi)}$ ) because, under the $N=n^{\mathcal{O}(1)}$ assumption,

$$
2^{\mathcal{O}\left(\operatorname{size}(p, \Phi)^{1+\frac{2}{D}}\right)} \leq 2^{\mathcal{O}\left(N^{2}\right)} \leq(q D)^{2^{\mathcal{O}(n)}} .
$$

(2) The above discussion assumes infinite precision. Under the presence of finite precision, the behaviors of Homology and CAD are radically different. We have already observed that Homology is numerically stable (and pointed the reader to [6, Section 7] for technical details). We can add some explanation here. The fundamental question in our context is the following: what is the finest precision required to ensure that the output of the algorithm is correct? No matter the algorithm, ill-posed inputs require infinite precision. And clearly, ill-conditioned inputs (i.e., those with a large condition number) will require a very large precision. But what about inputs with a moderate condition? The difference between Homology and CAD becomes critical here.

In a nutshell, CAD ends up performing computations with polynomials of doubly exponential degree. Round-off errors (even if they only occur when reading the input data) in the computations with these polynomials accumulate badly. In contrast, Homology performs an exponential number of computations, corresponding to the points in the grid, with polynomial-size objects. And these computations are performed independently of each other. This results in a very moderate accumulation of errors. Hence, while one can prove that the precision needed for a correct answer with Homology is small, this is certainly not the case with CAD. A recent result pointing in this direction is given in [13].

(3) The weak cost bounds in Theorem 1.1 depend on a choice of probability measure on the input space. Our choice (standard Gaussian in $\mathcal{P}_{\boldsymbol{d}}[q]$, or uniform in the sphere $\mathbb{S}\left(\mathcal{P}_{\boldsymbol{d}}[q]\right)$, w.r.t. the Weyl inner product) is the most common one for problems involving polynomial systems. But other choices are possible. In [7] a probabilistic analysis of $\kappa(f)$ in the case where $f$ is square ( $n$ polynomials in $n+1$ homogeneous variables) is done which is valid for a broad class of probability distributions. A weak cost analysis, now valid for all distributions in this class, of the algorithm for counting zeros of square systems in [5] follows. Such a result raises the following question.

Question 5.2. Can one develop a probabilistic analysis of $\bar{\kappa}_{\text {aff }}(p)$ for more general distributions? For the class of distributions in [7, this reduces to developing a probabilistic analysis of $\kappa(f)$ in the underdetermined case.

(4) Last but not least, it would be interesting to investigate the bit complexity of Hybrid. That is, to study whether weak cost bounds similar to those in Theorems 1.1 hold as well when the input is a tuple of integer polynomials. A particular case, with a clear interest, is that of the uniform distribution on the set $\{-M,-M+1,-M+2, \ldots, M\}$. A positive result for this case would turn Hybrid into a symbolic algorithm more efficient than CAD for tuples of integer polynomials. 


\section{References}

[1] P. Bürgisser, F. Cucker, and P. Lairez. Computing the homology of basic semialgebraic sets in weak exponential time. J. $A C M, 66(1): 5: 1-5: 30$, December 2018.

[2] P. Bürgisser, F. Cucker, and J. Tonelli-Cueto. Computing the Homology of Semialgebraic Sets. I: Lax Formulas. Foundations of Computational Mathematics, 20(1):71-118, 2020. On-line from May of 2019.

[3] P. Bürgisser and F. Cucker. Condition, volume 349 of Grundlehren der mathematischen Wissenschaften. Springer-Verlag, Berlin, 2013.

[4] G.E. Collins. Quantifier elimination for real closed fields by cylindrical algebraic decomposition, pages 134-183. Lecture Notes in Comput. Sci., Vol. 33. Springer, Berlin, 1975.

[5] F. Cucker, T. Krick, G. Malajovich, and M. Wschebor. A numerical algorithm for zero counting. I: Complexity and accuracy. J. Complexity, 24:582-605, 2008.

[6] F. Cucker, T. Krick, and M. Shub. Computing the Homology of Real Projective Sets. Found. Comput. Math., 18:929-970, 2018.

[7] A. Ergür, G. Paouris, and J.M. Rojas. Probabilistic condition number estimates for real polynomial systems I: A broader family of distributions. Found. Comput. Math., 19:131-157, 2019.

[8] A. Gabrielov and N. Vorobjov. Approximation of definable sets by compact families, and upper bounds on homotopy and homology. J. Lond. Math. Soc. (2), 80(1):35-54, 2009.

[9] C.G. Gibson, K. Wirthmüller, A.A. du Plessis, and E.J.N. Looijenga. Topological stability of smooth mappings. Lecture Notes in Mathematics, Vol. 552. Springer-Verlag, Berlin-New York, 1976.

[10] A. Hatcher. Algebraic topology. Cambridge University Press, Cambridge, 2002.

[11] J. Mather. Notes on topological stability. Bull. Amer. Math. Soc. (N.S.), 49(4):475-506, 2012.

[12] P. Niyogi, S. Smale, and S. Weinberger. Finding the homology of submanifolds with high confidence from random samples. Discrete Comput. Geom., 39(1-3):419-441, 2008.

[13] V. Noferini and A. Townsend. Numerical instability of resultant methods for multidimensional rootfinding. SIAM J. Numer. Anal., 54(2):719-743, 2016.

[14] J.T. Schwartz and M. Sharir. On the "piano movers" problem. II. General techniques for computing topological properties of real algebraic manifolds. Adv. in Appl. Math., 4(3):298$351,1983$.

[15] M. Shub and S. Smale. Complexity of Bézout's Theorem IV: probability of success; extensions. SIAM J. of Numer. Anal., 33:128-148, 1996.

[16] S. Smale. Newton's method estimates from data at one point. In R. Ewing, K. Gross, and C. Martin, editors, The Merging of Disciplines: New Directions in Pure, Applied, and Computational Mathematics, pages 185-196. Springer, 1986.

[17] J. Tonelli-Cueto. Condition and Homology in Semialgebraic Geometry. Doctoral thesis, Technische Universität Berlin, DepositOnce Repository, December 2019. http://dx.doi.org/10.14279/depositonce-9453

[18] H.R. Wüthrich. Ein Entscheidungsverfahren für die Theorie der reell-abgeschlossenen Körper. In E. Specker and V. Strassen, editors, Komplexität von Entscheidungsproblemen, volume 43 of Lect. Notes in Comp. Sci., pages 138-162. Springer-Verlag, 1976. 